\title{
PRIMAL-DUAL METHODS FOR THE COMPUTATION OF TRADING REGIONS UNDER PROPORTIONAL TRANSACTION COSTS
}
R. Herzog
K. Kunisch
J. Sass 
SFB sponsors:

- Austrian Science Fund (FWF)

- University of Graz

- Graz University of Technology

- Medical University of Graz

- Government of Styria

- City of Graz

Das Land

Steiermark

Stadt $\mathbf{G} \mathbf{R}$ A Z $\mathbf{Z}$ Wissenschaft 


\title{
PRIMAL-DUAL METHODS FOR THE COMPUTATION OF TRADING REGIONS UNDER PROPORTIONAL TRANSACTION COSTS
}

\author{
ROLAND HERZOG, KARL KUNISCH*, AND JÖRN SASS
}

\begin{abstract}
Portfolio optimization problems on a finite time horizon under proportional transaction costs are considered. The objective is to maximize the expected utility of the terminal wealth. The ensuing non-smooth timedependent Hamilton-Jacobi-Bellman (HJB) equation is solved by regularization and the application of a semi-smooth Newton method. Discretization in space is carried out by finite differences or finite elements. Computational results for one and two risky assets are provided.
\end{abstract}

\section{Introduction}

We consider numerical methods for the solution of a continuous-time portfolio optimization problem with a finite time horizon and under proportional transaction costs. An investor aims at maximizing the expected utility of the terminal value of the liquidated investment portfolio (wealth). The supremum of this expected value over all admissible trading strategies, the value function, satisfies a non-smooth time-dependent Hamilton-Jacobi-Bellman equation of the form

$$
\max \left\{V_{t}+\mathcal{L} V, \max _{1 \leq i \leq n} \mathcal{L}_{B_{i}} V, \max _{1 \leq i \leq n} \mathcal{L}_{S_{i}} V\right\}=0,
$$

cf. ? for a finite-time horizon and ??? for an optimization problem with infinite time horizon. The latter leads to a stationary problem (no dependency on time).

In our case, (1.1) is posed on the so-called solvency region $\mathcal{S}$ times the time interval $(0, T)$ and it is endowed with appropriate boundary and terminal conditions. The number $n$ denotes the number of risky assets (stocks) in the portfolio. While $\mathcal{L}$ is a second-order differential operator, the buy and sell operators $\mathcal{L}_{B_{i}}$ and $\mathcal{L}_{S_{i}}$ are of first order.

The sought-after optimal trading strategy is determined in terms of the timedependent partitioning of the solvency region $\mathcal{S}$ into subregions determined by which of the $2 n+1$ terms in (1.1) are zero (active), and by the manifolds separating these subregions. These subregions, the trading regions, describe which action is optimal, e.g., if the first term $V_{t}(y)+\mathcal{L} V(y)$ in (1.1) is zero for a risky fraction $y$, then it is optimal not to trade. The component $y_{i}$ of the risky fraction $y=\left(y_{1}, \ldots, y_{n}\right)^{\top}$ is the proportion of wealth invested in stock $i$. This is different from the corresponding problem without costs, for which ? showed that it is optimal to keep the risky fraction constant. This strategy requires continuous trading and cannot be followed when facing realistic transaction costs. Regions where one or several of the other operators in (1.1) are zero correspond to regions where

\footnotetext{
Date: April 13, 2011.

This research was carried out in part at the Johann Radon Institute for Computational and Applied Mathematics (RICAM), Austrian Academy of Sciences, Linz, Austria.

${ }^{*}$ Research supported in part by the Fonds zur Förderung der wissenschaftlichen Forschung (FWF) under SFB 32, Mathematical Optimization and Applications in the Biomedical Sciences.
} 
buying or selling a particular stock is optimal. For more references and other cost structures we refer to the introduction of ?. For our model with proportional costs these trading regions characterize the optimal strategy which is of the form that no trading occurs as long as the fractions invested in the stocks stay inside the no-trading (NT) region. Due to the dynamics of the stocks, these fractions might hit the boundary; then infinitesimal small trading to keep the fractions inside the NT-region is optimal, cf. [?, Section 9] for the stationary case.

It is the purpose of this paper to devise numerical methods for the solution of this problem, i.e. for finding the trading regions, on the basis of methods that were first analyzed for a simpler problem in ?. The main idea consists of replacing (1.1) by an approximating penalty-type formulation,

$$
V_{t}+\mathcal{L} V+c \sum_{i=1}^{n} \max \left\{0, \mathcal{L}_{B_{i}} V\right\}+c \sum_{i=1}^{n} \max \left\{0, \mathcal{L}_{S_{i}} V\right\}=0
$$

This approach was recently used in ? for a similar problem. For the simpler problem $\max \{-\triangle y+f,|\nabla y|-g\}=0$ it was shown in ? that the proposed penalty method is in fact regularizing and that the solutions of the penalized problems converge in appropriate function spaces to the solution of the original Hamilton-Jacobi-Bellman equation. After discretization in space and time, (1.2) can be solved using a semismooth Newton methods, see e.g. ?. The latter is naturally implemented in terms of an active set strategy.

Compared to the results in ?, in this paper we go a step beyond. In the case of one risky asset we compare the results obtained by applying a semi-smooth Newton method to both, the regularized and unregularized formulations of (1.2) to investigate the effect of the regularization parameter. In the two dimensional case, the use of regularization appears to be numerically essential. However, it may slow down the iteration procedure and give rise to highly convective contributions from the first-order operators $\mathcal{L}_{B_{i}}$ and $\mathcal{L}_{S_{i}}$. For this reason, in addition to using upwinding techniques, we propose to combine the Newton approach with an augmented Lagrangian concept. Differently from ?, we use a primal-dual strategy as opposed to a purely primal one for the determination of the active sets. A further distinctive feature of the proposed algorithm is an adaptive time-stepping technique.

We mention that a different numerical approach for the stationary problem with consumption as in ??? was proposed in ??. There the authors employ a monotonically decreasing update of the no-trading region, which is motivated by a policy improvement procedure. By contrast, for our time-dependent problem we propose a Newton scheme to resolve the trading and no-trading regions in every time step. We do not need a priori structural assumptions on the location of the NT region.

The contents of the paper are organized as follows. We summarize the necessary background on portfolio optimization in Section 2. In Section 3 we discuss and compare the regularized and unregularized approaches for the solution of (1.1) in the case of one risky asset $(n=1)$. Section 4 is devoted to the numerical treatment of the regularized problem for the case $n=2$. Numerical examples demonstrating the performance of the algorithms are provided for both cases.

\section{Background on Portfolio Optimization}

We consider a continuous-time market model consisting of one bond or bank account and $n \geq 1$ stocks with prices $\left(P_{0}(t)\right)_{t \in[0, T]}$ and $\left(P_{i}(t)\right)_{t \in[0, T]}, i=1, \ldots, n$, 
respectively. For given interest rate $r \geq 0$, trend parameter $\mu \in \mathbb{R}^{n}$, and nonsingular volatility-matrix $\sigma \in \mathbb{R}^{n \times n}$, these evolve according to

$$
\begin{array}{lll}
\mathrm{d} P_{0}(t)=P_{0}(t) r \mathrm{~d} t, & P_{0}(0)=1, & \\
\mathrm{~d} P_{i}(t)=P_{i}(t) \mu_{i} \mathrm{~d} t+P_{i}(t) \sum_{j=1}^{n} \sigma_{i j} \mathrm{~d} W_{j}(t), & P_{i}(0)=1, & i=1, \ldots, n .
\end{array}
$$

Here $W=(W(t))_{t \in[0, T]}$ is an $n$-dimensional standard Brownian motion on a probability space $(\Omega, \mathcal{A}, P)$. Let $\mathcal{F}=\left(\mathcal{F}_{t}\right)_{t \in[0, T]}$ denote the augmented filtration generated by $W$.

2.1. Trading Without Transaction Costs. Without transaction costs, the trading of an investor may be described by initial capital $\bar{X}(0)>0$ and risky fraction process $(\eta(t))_{t \in[0, T]}, \eta(t)=\left(\eta_{1}(t), \ldots, \eta_{n}(t)\right)^{\top}$, where $\eta_{i}(t)$ is the fraction of the portfolio value (wealth) which is held in stock $i$ at time $t$. The corresponding wealth process $(\bar{X}(t))_{t \in[0, T]}$ is defined self-financing by

$$
\mathrm{d} \bar{X}(t)=\left(1-\mathbf{1}^{\top} \eta(t)\right) \bar{X}(t) r \mathrm{~d} t+\sum_{i=1}^{n} \eta_{i}(t) \bar{X}(t)\left(\mu_{i} \mathrm{~d} t+\sum_{j=1}^{n} \sigma_{i j} \mathrm{~d} W_{j}(t)\right),
$$

where $\mathbf{1}=(1, \ldots, 1)^{\top}$. In this section we call $\left(\eta_{t}\right)_{t \geq 0}$ admissible if it is adapted, measurable, bounded, and $\bar{X}(T)>0$ holds. The terminal wealth $x=\bar{X}(T)>0$ is evaluated by a power utility function

$$
\frac{1}{\alpha} x^{\alpha} \quad \text { for any } \quad \alpha<1, \quad \alpha \neq 0 .
$$

The parameter $\alpha$ models the preferences of an investor. The strategy for the limiting case $\alpha \rightarrow 0$ corresponds to logarithmic utility, i.e., to maximizing the expected rate of return. The case $\alpha>0$ corresponds to less risk averse and $\alpha<0$ to more risk averse utility functions. ? showed that for logarithmic $(\alpha=0)$ and power utility the optimal trading strategy is given by a constant optimal risky fraction

$$
\eta(t)=\hat{\eta}, \quad t \in[0, T], \quad \text { for } \quad \hat{\eta}=\frac{1}{1-\alpha} A^{-1}(\mu-r \mathbf{1}),
$$

where $A=\sigma \sigma^{\top}$ denotes the covariance matrix of the stock returns.

2.2. Proportional Transaction Costs. Keeping the risky fraction constant like in (2.2) involves continuous trading. This is no longer adequate in the presence of transaction costs. We consider proportional costs $\gamma \in(0,1)$ which correspond to the proportion of the traded volume which has to be paid as fees. These are paid from the bank account (bond).

The trading policy can be described by increasing processes $L_{i}=\left(L_{i}(t)\right)_{t \in[0, T]}$ and $M_{i}=\left(M_{i}(t)\right)_{t \in[0, T]}$ representing the cumulative purchases and sales of stock $i$, $i=1, \ldots, n$. We require that $L=\left(L_{1}, \ldots, L_{n}\right)^{\top}, M=\left(M_{1}, \ldots, M_{n}\right)^{\top}$ are rightcontinuous, $\mathcal{F}$-adapted, with initial values $L(0-)=M(0-)=0$. Since transaction fees are paid from the bank account, the dynamics of the controlled wealth processes $\left(X_{0}(t)\right)_{t \in[0, T]}$ and $\left(X_{i}(t)\right)_{t \in[0, T]}$, corresponding to the amount of money in the bank account and the amount invested in stock $i$, are

$$
\begin{aligned}
& \mathrm{d} X_{0}(t)=r X_{0}(t) \mathrm{d} t-(1+\gamma) \mathrm{d}\left(\mathbf{1}^{\top} L(t)\right)+(1-\gamma) \mathrm{d}\left(\mathbf{1}^{\top} M(t)\right) \\
& \mathrm{d} X_{i}(t)=X_{i}(t) \mu_{i} \mathrm{~d} t+X_{i}(t) \sum_{j=1}^{n} \sigma_{i j} \mathrm{~d} W_{j}(t)+\mathrm{d} L_{i}(t)-\mathrm{d} M_{i}(t)
\end{aligned}
$$

We write the wealth in stocks as vector $X(t)=\left(X_{1}(t), \ldots, X_{n}(t)\right)^{\top}$. 
The objective is the maximization of the expected utility at the terminal trading time $T$, over all control processes $L$ and $M$ which satisfy the conditions above and for which the wealth processes $X_{i}, i=0, \ldots, n$, stay in the solvency region

$$
\mathcal{S}^{0}:=\left\{\left(x_{0}, x\right): x_{0} \in \mathbb{R}, x \in \mathbb{R}^{n}, x_{0}+\mathbf{1}^{\top} x-\gamma\|x\|_{1}>0\right\}
$$

which consists of all positions in bond and stocks for which a strictly positive wealth remains after liquidating the stock holdings. Note that in (2.4), $\gamma\|x\|_{1}=$ $\sum_{i=1}^{n} \gamma\left|x_{i}\right|$ are the liquidation costs. Thus we require

$$
\left(X_{0}(t), X(t)\right) \in \mathcal{S}^{0}, \quad t \in[0, T) .
$$

Suppose that $\left(X_{0}(0-), X_{1}(0-), \ldots, X_{n}(0-)\right) \in \mathcal{S}^{0}$. Accordingly, we consider the maximization of the expected utility of the terminal total wealth after liquidating the position in the stocks, i.e., we consider the value function

$$
\begin{aligned}
& \Phi\left(t, x_{0}, x\right) \\
& =\sup _{(L, M)} \mathrm{E}\left[\frac{1}{\alpha}\left(X_{0}(T)+\mathbf{1}^{\top} X(T)-\gamma\|X(T)\|_{1}\right)^{\alpha} \mid X_{0}(t-)=x_{0}, X(t-)=x\right] .
\end{aligned}
$$

Alternatively, we might look at maximizing the expected utility of the terminal total wealth, cf. Remark 2.3 below.

Theorem 2.1. The value function $\Phi$ is continuous, concave in $\left(x_{0}, x\right)$, and satisfies the homotheticity property

$$
\Phi\left(t, c x_{0}, c x\right)=c^{\alpha} \Phi\left(t, x_{0}, x\right) \quad \text { for } \quad c>0, t \in[0, T),\left(x_{0}, x\right) \in \mathcal{S}^{0},
$$

as well as the boundary conditions

$$
\Phi\left(t, x_{0}, x\right) \rightarrow\left\{\begin{array}{ll}
0, & \text { if } 0<\alpha<1, \\
-\infty, & \text { if } \alpha<0 .
\end{array} \quad \text { for }\left(x_{0}, x\right) \rightarrow\left(\bar{x}_{0}, \bar{x}\right) \in \partial \mathcal{S}^{0} .\right.
$$

Further, $\Phi$ is a viscosity solution of

$$
\begin{array}{ll}
\max \left\{\Phi_{t}+\mathcal{A} \Phi, \max _{1 \leq i \leq n} \mathcal{A}_{B_{i}} \Phi, \max _{1 \leq i \leq n} \mathcal{A}_{S_{i}} \Phi\right\}=0 & \text { on }[0, T) \times \mathcal{S}^{0}, \\
\Phi\left(T, x_{0}, x\right)=\frac{1}{\alpha}\left(x_{0}+\mathbf{1}^{\top} x-\gamma\|x\|_{1}\right)^{\alpha} & \text { for }\left(x_{0}, x\right) \in \mathcal{S}^{0},
\end{array}
$$

where the differential operators $\mathcal{A}$ (generator of $\left(X_{0}, X\right)$ ) and $\mathcal{A}_{B_{i}}, \mathcal{A}_{S_{i}}, i=1, \ldots, n$, are defined by

$$
\begin{aligned}
& \mathcal{A} h\left(x_{0}, x\right)=r x_{0} h_{x_{0}}\left(x_{0}, x\right)+\sum_{i=1}^{n} \mu_{i} x_{i} h_{x_{i}}\left(x_{0}, x\right)+\frac{1}{2} \sum_{i, j=1}^{n} A_{i, j} x_{i} x_{j} h_{x_{i}, x_{j}}\left(x_{0}, x\right), \\
& \mathcal{A}_{B_{i}} h\left(x_{0}, x\right)=-(1+\gamma) h_{x_{0}}\left(x_{0}, x\right)+h_{x_{i}}\left(x_{0}, x\right), \\
& \mathcal{A}_{S_{i}} h\left(x_{0}, x\right)=(1-\gamma) h_{x_{0}}\left(x_{0}, x\right)-h_{x_{i}}\left(x_{0}, x\right)
\end{aligned}
$$

for all smooth functions $h$.

Proof. The proof can be carried out similarly as in ? or ?, even if we consider a finite time horizon problem and consequently a time-dependent value function. In particular the first part is standard, corresponding to [?, Propositions 3.1-3.3]. Continuity of $\Phi$ in $t$ can be shown using the no-trading strategy and the optimal strategy without costs to derive lower and upper bounds for the change of the value function in time. The convergence in (2.7) follows from arguments as used in [?, Corollaries 5.5, 5.8] and the fact that $\Phi$ is a viscosity solution follows along the lines of [?, Sections 6], only that we need the continuity of $\Phi$ in $t$ as well. 
Depending on which of the three terms in the outer max operation in (2.8) is active at a given $\left(t, x_{0}, x\right) \in[0, T] \times \mathcal{S}^{0}$, i.e., equals 0 , we say that $\left(t, x_{0}, x\right)$ belongs to the no-trading region, or the buy or sell region, respectively. More precisely, at time $t$, we define the buy regions, sell regions and the no-trading region as follows:

$$
\begin{aligned}
B_{i}^{0}(t) & =\left\{\left(x_{0}, x\right) \in \mathcal{S}^{0}: \mathcal{A}_{B_{i}} \Phi\left(t, x_{0}, x\right)=0\right\}, \\
S_{i}^{0}(t) & =\left\{\left(x_{0}, x\right) \in \mathcal{S}^{0}: \mathcal{A}_{S_{i}} \Phi\left(t, x_{0}, x_{1}\right)=0\right\}, \\
N T^{0}(t) & =\mathcal{S}^{0} \backslash \bigcup_{1 \leq i \leq n}\left(B_{i}^{0}(t) \cup S_{i}^{0}(t)\right) .
\end{aligned}
$$

The boundaries between these sets determine the optimal trading policy.

Based on Theorem 2.1 we may further analyze the regularity of the value function and the properties of the trading regions. Since the arguments differ from those in ? due to the finite time horizon (dependency on time) and since we consider $n \geq 1$ stocks, we state the corrsponding results as an assumption. These claims are supported by the results in ? and the numerical analysis in ???. The numerical results in this paper may give rise to derive further properties of the trading regions which allow for a more direct proof.

Assumption 2.2. The value function $\Phi=\Phi\left(t, x_{0}, x\right)$ is continuously differentiable in $t$, continuously differentiable in $x_{0}$ and twice continuously differentiable in $x=\left(x_{1}, \ldots, x_{n}\right)^{\top}$ for $\left(t, x_{0}, x\right) \in(0, T) \times\left(\mathcal{S}^{0} \backslash\left\{\left(x_{0}, x_{1}, \ldots x_{n}\right) \mid x_{i}=0\right.\right.$ for some $\left.\left.i\right\}\right)$. Further, $N T^{0}(t) \neq \emptyset$ for all $t \in[0, T)$.

Remark 2.3. Instead of $\Phi$ we might also consider

$$
\tilde{\Phi}\left(t, x_{0}, x\right)=\sup _{(L, M)} \mathrm{E}\left[\frac{1}{\alpha}\left(X_{0}(T)+\mathbf{1}^{\top} X(T)\right)^{\alpha} \mid X_{0}(t-)=x_{0}, X(t-)=x\right]
$$

which corresponds to maximizing the expected utility of the terminal total wealth (i.e., without liquidating the position in the stocks). In Theorem 2.1 this would change only the terminal condition to $\tilde{\Phi}\left(T, x_{0}, x_{1}\right)=\frac{1}{\alpha}\left(x_{0}+\mathbf{1}^{\top} x\right)^{\alpha}$ instead of (2.9).

Remark 2.4. If neither short selling nor borrowing are allowed, we would require that the wealth processes $X_{i}, i=0, \ldots, n$, stay positive and the total wealth stays strictly positive, i.e.,

$$
\left(X_{0}(t), X(t)\right) \in \mathcal{D}^{0}:=\mathbb{R}_{+}^{n+1} \backslash\{\mathbf{0}\} \quad \text { for } t \in[0, T] .
$$

While the operators have the same form, not all actions (selling or buying stock i) are admissible on the boundary of $\mathcal{D}^{0}$, which restricts the number of operators in (2.8). In ? a result corresponding to Theorem 2.1 is derived. The numerical treatment is possible with the methods developed in this paper.

2.3. Reduction of the Dimension. It is convenient to consider the risky fraction process rather than the wealth process (2.3). For wealth $x_{i}$ in stock $i$ and total wealth $\xi=x_{0}+\mathbf{1}^{\top} x$, the risky fractions are given by $y_{i}=\frac{x_{i}}{\xi}, i=1, \ldots, n$.

From the homotheticity property (2.6) of $\Phi$ we deduce

$$
\Phi\left(t, x_{0}, x\right)=\xi^{\alpha} \Phi\left(t, \frac{x_{0}}{\xi}, \frac{x_{1}}{\xi}, \ldots, \frac{x_{n}}{\xi}\right) .
$$

Thus it is enough to consider

$$
V(t, y)=\Phi\left(t, 1-\mathbf{1}^{\top} y, y\right), \quad y \in \mathcal{S}
$$

where

$$
\mathcal{S}=\left\{y \in \mathbb{R}^{n}: \gamma\|y\|_{1}<1\right\}
$$


is the solvency region in terms of the risky fractions. For $\Phi\left(t, x_{0}, x\right)=\xi^{\alpha} V(t, x / \xi)$, we obtain

$$
\begin{aligned}
& \Phi_{t}=\xi^{\alpha} V_{t}, \quad \Phi_{x_{0}}=\xi^{\alpha-1}\left(\alpha V-y^{\top} V_{y}\right), \quad \Phi_{x_{i}}=\xi^{\alpha-1}\left(\alpha V+\left(\mathrm{e}_{i}-y\right)^{\top} V_{y}\right), \\
& \Phi_{x_{i}, x_{j}}=\xi^{\alpha-2}\left(\alpha(1-\alpha) V+(\alpha-1)\left(\mathrm{e}_{i}+e_{j}-2 y\right)^{\top} V_{y}+\left(\mathrm{e}_{i}-y\right)^{\top} V_{y y}\left(e_{j}-y\right)\right),
\end{aligned}
$$

where $V_{y}$ and $V_{y y}$ denote the gradient and the Hessian of $V$, and $\mathrm{e}_{1}, \ldots, \mathrm{e}_{n}$ are the unit vectors in $\mathbb{R}^{n}$. We get for $y=x / \xi$

$$
\begin{aligned}
& \Phi_{t}\left(t, x_{0}, x\right)=\xi^{\alpha} V_{t}(t, y), \quad \mathcal{A} \Phi\left(t, x_{0}, x\right)=\xi^{\alpha} \mathcal{L} V(t, y), \\
& \mathcal{A}_{B_{i}} \Phi\left(t, x_{0}, x\right)=\xi^{\alpha-1} \mathcal{L}_{B_{i}}(t, y) V, \quad \mathcal{A}_{S_{i}} \Phi\left(t, x_{0}, x\right)=\xi^{\alpha-1} \mathcal{L}_{S_{i}} V(t, y) .
\end{aligned}
$$

For classical solutions for which the above derivatives exist and are continuous, Theorem 2.1 carries over directly to Theorem 2.5 below. For a viscosity solution $\Phi$ it can be shown that also $V$ is a viscosity solution of (2.15), which then yields Theorem 2.5, cf. [?, Proposition 8.1]:

Theorem 2.5. The value function $V$ is continuous and concave in $y$ with

$$
V(t, y) \rightarrow\left\{\begin{array}{ll}
0, & \text { if } 0<\alpha<1 \\
-\infty, & \text { if } \alpha<0
\end{array} \quad \text { for } y \rightarrow \bar{y} \in \partial \mathcal{S}, \quad t \in(0, T) .\right.
$$

Further, $V$ is a viscosity solution of

$$
\max \left\{V_{t}+\mathcal{L} V, \max _{1 \leq i \leq n} \mathcal{L}_{B_{i}} V, \max _{1 \leq i \leq n} \mathcal{L}_{S_{i}} V\right\}=0
$$

on $[0, T) \times \mathcal{S}$ with terminal condition

$$
V(T, y)=\frac{1}{\alpha}\left(1-\gamma\|y\|_{1}\right)^{\alpha}, \quad y \in \mathcal{S} .
$$

The operators in (2.15) are defined by

$$
\begin{aligned}
\mathcal{L} V & =\alpha\left(r+(\mu-r \mathbf{1})^{\top} y-\frac{1}{2}(1-\alpha) y^{\top} A y\right) V \\
& +\left[\left(\operatorname{diag}(\mu-r \mathbf{1})-(\mu-r \mathbf{1})^{\top} y I\right) y-\frac{1}{2}(1-\alpha) \sum_{i, j=1}^{n} A_{i j} y_{i} y_{j}\left(\mathrm{e}_{i}+\mathrm{e}_{j}-2 y\right)\right]^{\top} V_{y} \\
& +\frac{1}{2} \sum_{i, j=1}^{n} A_{i j} y_{i} y_{j}\left(\mathrm{e}_{i}-y\right)^{\top} V_{y y}\left(\mathrm{e}_{j}-y\right), \\
\mathcal{L}_{B_{i}} V & =-\alpha \gamma V+\left(\mathrm{e}_{i}+\gamma y\right)^{\top} V_{y}, \quad i=1, \ldots, n \\
\mathcal{L}_{S_{i}} V & =-\alpha \gamma V-\left(\mathrm{e}_{i}-\gamma y\right)^{\top} V_{y}, \quad i=1, \ldots, n .
\end{aligned}
$$

The trading regions are now given by

$$
\begin{aligned}
B_{i}(t) & =\left\{y \in \mathcal{S}: \mathcal{L}_{B_{i}} V(t, y)=0\right\}, \\
S_{i}(t) & =\left\{y \in \mathcal{S}: \mathcal{L}_{S_{i}} V(t, y)=0\right\}, \\
N T(t) & =\mathcal{S} \backslash \bigcup_{1 \leq i \leq n}\left(B_{i}(t) \cup S_{i}(t)\right) .
\end{aligned}
$$

They correspond to buying stock $i$, selling stock $i$ and not trading at all. On

$$
\bigcap_{1 \leq i \leq n} R_{i}, \quad R_{i} \in\left\{B_{i}, S_{i}\right\}
$$

we thus get from $\mathcal{L}_{R_{i}} V=0, i=1, \ldots, n$, that

$$
V(t, y)=C_{R_{1}, \ldots, R_{n}}(t)\left(1+\sum_{i=1}^{n} \gamma_{i} y_{i}\right)^{\alpha}
$$

where $\gamma_{i}=\gamma$ if $R_{i}=B_{i}$ and $\gamma_{i}=-\gamma$ if $R_{i}=S_{i}$. 
Remark 2.6. (1) Assumption 2.2 implies that $V(t, \cdot)$ is twice continuously differentiable on $\mathcal{S} \backslash \partial \mathcal{D}$ where $\mathcal{D}$ is the simplex in $\mathbb{R}^{n}$. Due to the homotheticity property (2.6) the trading regions for the original problem are cones. Therefore, $N T^{0}(t) \neq \emptyset$ implies also $N T(t) \neq \emptyset$ for all $t \in[0, T)$.

(2) The homotheticity property (2.11) holds also for the value function $\tilde{\Phi}$ in (2.10) without terminal liquidation, see Remark 2.3. Hence we can consider $\tilde{V}(t, y)=\tilde{\Phi}\left(t, 1-\mathbf{1}^{\top} y, y\right)$ for which we have to solve $(2.15)$ with terminal condition $\tilde{V}(T, y)=1 / \alpha$.

(3) In the case that borrowing $\left(\mathbf{1}^{\top} y>1\right)$ and short selling $\left(y_{i}<0\right)$ are not allowed, after reducing the dimension we have to solve on $[0, T) \times \mathcal{D}$ a Hamilton-Jacobi-Bellman (HJB) equation based on the same operators as in Theorem 2.5 and using the same terminal condition (2.16). Here $\mathcal{D}$ denotes the simplex in $\mathbb{R}^{n}$. On the boundary $\partial \mathcal{D}$ we have to take care which actions are not admissible and may have to exclude the corresponding inequalities, cf. ?. For this problem with no borrowing and no short selling, some of $B_{i}(t)$ or $S_{i}(t)$ may be empty. If that happens we need boundary conditions different from those given by $\mathcal{L}_{B_{i}} V=0, \mathcal{L}_{S_{i}} V=0$ on the boundary of $N T(t)$. In this case these boundary conditions follow from requiring $V_{t}+\mathcal{L} V=0$ on the boundary.

(4) While all examples in this paper use symmetric proportional trading costs $\gamma$, our approach can be easily extended to cover the case of different costs for buying and selling, which may be different for each asset. Analogous results can be obtained for logarithmic utility.

2.4. Numerical Treatment of Portfolio Optimization as a Non-Variational Complementarity Problem. It should be recognized that, even in the stationary case with $V_{t}$ not appearing in (2.15), this problem is not of variational type. It is thus distinct from the obstacle problem, not only since the constraints involve spatial derivatives of the state, rather than the state itself, but also since it cannot be interpreted in a straightforward way as the necessary optimality condition of a minimization problem in function space. In a function space setting such problems have received very little attention. In ? we investigated

$$
\begin{aligned}
& \max \{-\triangle u-f, \quad|\nabla u|-g\}=0 \quad \text { a.e. in } \Omega, \\
& u=0 \quad \text { on } \Gamma=\partial \Omega,
\end{aligned}
$$

where $\Omega \subset \mathbb{R}^{d}$ is a bounded domain with smooth boundary $\Gamma$, as a prerequisite to the present work. There we mainly investigated two approaches, namely of (3.6) as a complementarity problem, which involves the introduction of a new variable $\lambda$ for the term $|\nabla u|-g$, and alternatively a regularization procedure, which realizes the constraint on $|\nabla u|-g$ by means of a penalty formulation. The complementarity approach was already treated in ? for the one dimensional case of (2.15). The introduction of the regularization term allows to interpret the approach as a semismooth Newton method in function spaces, whereas without the regularization the interpretation as semi-smooth Newton method is only possible after discretization. This is described in more detail in section below.

The regularization approach presented from the point of view of penalization was recently also used in ?. In this paper we go a step beyond. In the one dimensional case we compare the results between the regularization and the complementarity formulation to investigate the effect of the regularization parameter. In the two dimensional case, the use of regularization appears to be numerically essential, on the other hand it may slow down the iteration procedure and influence the condition number of the system matrix. For this reason we propose to combine the 
Newton approach that is used to solve the regularized problems with an augmented Lagrangian concept. This involves the interpretation of $\lambda$ as a Lagrange multiplier and updating it according to a multiplier scheme well known from numerical realization of inequality constraints, ??. Differently from ?, we use a primal-dual strategy as opposed to a purely primal one for the determination of the active sets. A further distinctive feature of the proposed algorithm is an adaptive time-stepping technique.

\section{The Case of One Risky Asset}

In the case of only one risky asset (stock) $n=1$, equation (2.15) reduces to

$$
\max \left\{V_{t}+\mathcal{L} V, \mathcal{L}_{B} V, \mathcal{L}_{S} V\right\}=0 \quad \text { on }(0, T) \times \mathcal{S}
$$

on $\mathcal{S}=(-1 / \gamma, 1 / \gamma)$ with boundary conditions

$$
V(t,-1 / \gamma)=V(t, 1 / \gamma)=\left\{\begin{array}{ll}
0, & \text { if } 0<\alpha<1 \\
-\infty, & \text { if } \alpha<0
\end{array} \quad \text { for all } t \in(0, T)\right.
$$

and terminal conditions on $\mathcal{S}$

$$
\begin{aligned}
& V(T, y)=\frac{1}{\alpha} \quad \text { without liquidation costs } \\
& V(T, y)=\frac{1}{\alpha}\left(1-\gamma\|y\|_{1}\right)^{\alpha} \quad \text { with liquidation costs. }
\end{aligned}
$$

By (2.17), the linear differential operators in (3.1) become

$$
\begin{aligned}
\mathcal{L} V= & {\left[\alpha(r+(\mu-r) y)+\frac{1}{2} \alpha(\alpha-1) \sigma^{2} y^{2}\right] V } \\
& +\left[(\mu-r) y(1-y)+(\alpha-1) \sigma^{2} y^{2}(1-y)\right] V_{y}+\frac{1}{2} \sigma^{2} y^{2}(1-y)^{2} V_{y y} \\
\mathcal{L}_{B} V= & (1+\gamma y) V_{y}-\alpha \gamma V \\
\mathcal{L}_{S} V= & -(1-\gamma y) V_{y}-\alpha \gamma V .
\end{aligned}
$$

3.1. Regularization. Instead of treating (3.1)-(3.3) directly, we consider the regularized formulation

$$
V_{t}+\mathcal{L} V+c \max \left\{0, \mathcal{L}_{B} V\right\}+c \max \left\{0, \mathcal{L}_{S} V\right\}=0
$$

with regularization parameter $c>0$ and subject to the boundary and terminal conditions (3.2)-(3.3). The motivation for introducing this formulation for the numerical realization of (3.1) resides in the fact that we shall utilize a Newton type method. Clearly this is impeded by the max-operation appearing in (3.1). The regularized form (3.5), however, lends itself to a semi-smooth Newton treatment. Let us explain this point in some detail for a significantly simpler problem which, however, still contains the essential feature of (3.5). For this purpose we consider

$$
\begin{aligned}
& \max \left\{-\Delta u-f, \quad u^{\prime}-g, \quad h-u^{\prime}\right\}=0 \quad \text { a.e. in } \Omega, \\
& u=0 \quad \text { on } \partial \Omega,
\end{aligned}
$$

where $\Omega$ is an interval in $\mathbb{R}$, and $f, g$ and $h$, with $g \geq h$ are given. Just as the full problem (3.1) our model problem involves the max operation over a second-order diffusion process and inequality constraints on first order terms of the unknown function $u$.

Instead of (3.6), we consider a sequence of regularized problems for an increasing sequence of parameters $c>0$ :

$$
-\triangle u+c \max \left\{0, u^{\prime}-g\right\}+c \max \left\{0, h-u^{\prime}\right\}=f \quad \text { in } \Omega, \quad u=0 \quad \text { on } \partial \Omega .
$$

On the inactive set $\left\{h>u^{\prime}\right\} \cap\left\{u^{\prime}<g\right\}$ we have $-\triangle u=f$, just as in (3.6), whereas diffusion is added on the active set $\left\{h \leq u^{\prime}\right\} \cup\left\{u^{\prime} \geq g\right\}$. This justifies calling (3.7) 
a regularization of (3.6). In (3.7) only lower order terms appear under the max operation. It is therefore quite straightforward to argue that

$$
F(\lambda)=\lambda-c \max \left\{0,\left((-\triangle)^{-1}(f-\lambda)\right)^{\prime}-g\right\}-c \max \left\{0, h-\left((-\triangle)^{-1}(f-\lambda)\right)^{\prime}\right\}
$$

is Newton differentiable, from $L^{2}(\Omega)$ to itsself, and consequently the semi-smooth Newton algorithm applied to $F(\lambda)=0$ converges locally $q$-superlinearly to the solution $u_{c}$ of (3.7), see ??, for example. Above $\triangle^{-1}$ denotes the solution operator for the Laplacian in $\Omega$ with homogenous Dirichlet boundary conditions. To describe the asymptotic behavior, we set

$$
\lambda_{c}^{+}:=c \max \left\{0, u_{c}^{\prime}-g\right\}, \quad \lambda_{c}^{-}:=c \max \left\{0, h-u_{c}^{\prime}\right\}, \quad \lambda_{c}:=\lambda_{c}^{+}+\lambda_{c}^{-} .
$$

Then, as $c \rightarrow \infty$ the pair $\left(u_{c}, \lambda_{c}\right)$ converges to the solution of

$$
\begin{array}{rrrrr} 
& & -\triangle u+\lambda^{+}+\lambda^{-}=f & \text { a.e. in } \Omega \\
\lambda^{+} \geq 0, & u^{\prime}-g \leq 0, & \lambda^{+}\left(u^{\prime}-g\right)=0 & \text { a.e. in } \Omega \\
\lambda^{-} \geq 0, & -u^{\prime}+h \leq 0, & \lambda^{-}\left(-u^{\prime}+h\right)=0 & \text { a.e. in } \Omega,
\end{array}
$$

which is the complementarity formulation of (3.6).

The second and third equations in (3.10) can equivalently be expressed as

$$
\lambda^{+}=\max \left(0, \lambda^{+}+\varrho\left(u^{\prime}-g\right)\right), \quad \lambda^{-}=\max \left(0, \lambda^{-}+\varrho\left(h-u^{\prime}\right)\right),
$$

for any fixed $\varrho>0$, i.e. $\varrho$ is not a regularization parameter. Exploiting the fact that the upper and lower bounds cannot be active simultaneously if $g>h$, system (3.6) can equivalently be expressed as

$$
\begin{aligned}
\tilde{F}(\lambda)=\lambda-\max \left\{0, \lambda+\varrho\left((-\triangle)^{-1}(f-\lambda)\right)^{\prime}-g\right\} & \\
& -\max \left\{0, \lambda+\varrho\left(h-(-\triangle)^{-1}(f-\lambda)\right)^{\prime}\right\},
\end{aligned}
$$

where the relation to $\lambda^{ \pm}$is given by $\lambda=\lambda^{+}$on the set $\left\{u^{\prime}=g\right\}, \lambda=\lambda^{-}$on $\left\{u^{\prime}=h\right\}$, and $\lambda=\lambda^{ \pm}=0$ on the inactive set. The max-operations appearing in (3.8) and (3.12) are distinctly different. In the former, the unknown quantity $\lambda$ appears with a smoothing operation $\triangle^{-1}$, whereas in the latter it stands by itself. As a consequence $F$ is Newton differentiable whereas $\tilde{F}$ is not. We refer to ? for the precise definition of Newton differentiability. Here it suffices to recall that $\max \{0, \cdot\}: L^{r}(\Omega) \rightarrow L^{2}(\Omega)$ is Newton differentiable (with generalized derivative given by an indicator function) if and only if $r>2$. This can be used to argue Newton differentiability of $F$, for any $c>0$. This also gives another justification to refer to (3.7) as a regularization of (3.6). Analogous results as discussed here for the one-dimensional case also hold in higher dimensions ?. There it was also shown that the solutions to the regularized problem are $W^{2, p}(\Omega)$ regular, for any $p \in[1, \infty)$, whereas the solution to the multi-dimensional analog of (3.6) are in $H^{2}(\Omega)$.

Clearly, the choice of the regularization parameter $c$ must be addressed. In practice this will frequently be done heuristically, but for the related class of optimal control problems with pointwise constraints, path-following algorithms were developed in ? which allow self-tuning of the regularization parameter $c$.

3.2. Discretization in Time. For the numerical realization a semi-discretization of (3.5) backwards in time by the one-step $\theta$ method with $\theta \in(0,1]$ is used. Let $V^{n}$ denote the unknown at time level $n$. Then $V^{n}$ is computed from $V^{n+1}$ according 
to

$$
\begin{aligned}
& \frac{V^{n+1}-V^{n}}{\tau}+\mathcal{L}\left(\theta V^{n}+(1-\theta)\right.\left.V^{n+1}\right)+c \max \left\{0, \mathcal{L}_{B}\left(\theta V^{n}+(1-\theta) V^{n+1}\right)\right\} \\
&+c \max \left\{0, \mathcal{L}_{S}\left(\theta V^{n}+(1-\theta) V^{n+1}\right)\right\}=0
\end{aligned}
$$

subject to the boundary conditions (3.2). The time step size is denoted by $\tau$. For the specific choices $\theta=1$ and $\theta=1 / 2$ the scheme becomes the implicit (backward) Euler and the Crank-Nicolson methods, respectively.

3.3. Semi-Smooth Newton Method. At any given time level $n$, (3.13) represents a second-order elliptic partial differential equation for the variable $V^{n}$ with a non-smooth first-order term. The nonsmoothness arises from the presence of the $\max \{0, \cdot\}$ operation. However, this operation enjoys the Newton differentiability property, which allows for the formulation of a generalized Newton's method. A Newton derivative of $\max \left\{0, \mathcal{L}_{B} V\right\}$ is given by the indicator function of $\left\{\mathcal{L}_{B} V>0\right\}$. The structure of the indicator function entails that the Newton step takes the form of an active set method. This has been rigorously proved in ? for a similar problem setting.

Apart from the $\max \{0, \cdot\}$ terms, (3.13) is a linear equation. Hence the Newton step, written in terms of the new iterate $V=V_{k+1}^{n}$ is given by

$$
\begin{aligned}
\frac{V^{n+1}-V}{\tau}+\mathcal{L}\left(\theta V+(1-\theta) V^{n+1}\right) & +c \chi_{A_{k}^{B}} \mathcal{L}_{B}\left(\theta V+(1-\theta) V^{n+1}\right) \\
& +c \chi_{A_{k}^{S}} \mathcal{L}_{S}\left(\theta V+(1-\theta) V^{n+1}\right)=0,
\end{aligned}
$$

subject to the boundary conditions (3.2), where

$$
\begin{aligned}
& A_{k}^{B}=\left\{y \in(-1 / \gamma, 1 / \gamma): \mathcal{L}_{B}\left(\theta V_{k}^{n}+(1-\theta) V^{n+1}\right)>0\right\} \\
& A_{k}^{S}=\left\{y \in(-1 / \gamma, 1 / \gamma): \mathcal{L}_{S}\left(\theta V_{k}^{n}+(1-\theta) V^{n+1}\right)>0\right\} .
\end{aligned}
$$

The complete semi-smooth Newton (SSN) algorithm is given as Algorithm 3.1. An initial guess for $V_{0}^{n}$ on the current time level is obtained from linear extrapolation of $V^{n+1}$ and $V^{n+2}$, i.e.,

$$
V_{0}^{n}=2 V^{n+1}-V^{n+2},
$$

or $V_{0}^{N-1}=V^{N}$ in case of the first time step.

3.4. Discretization in Space and Treatment of Boundary Conditions. The spatial operators in (3.14)-(3.15) are discretized by finite differences. The trading bounds $\sup B(t)$ and inf $S(t)$ lie to the left and right of the Merton fraction, see $(2.2)$,

$$
\hat{\eta}=\frac{1}{1-\alpha} \frac{\mu-r}{\sigma^{2}}
$$

In order to resolve the trading bounds accurately, a refined grid in $[\hat{\eta}-1, \hat{\eta}+1]$ around the Merton fraction and a coarse grid away from it on $[-1 / \gamma, \hat{\eta}-1]$ and $[\hat{\eta}+1,1 / \gamma]$ was used. For $\hat{\eta} \in(0,1)$ this choice guarantees that the $N T$-region is covered by the refined grid. For $\hat{\eta}$ far away from 0 it might happen that it does not lie in the NT-region, see e.g., ??, and the choice for the grid would have to be adapted.

The second derivative $V_{y y}$ was discretized by the standard stencil $\left[\begin{array}{lll}1 & -2 & 1\end{array}\right] / h^{2}$. The convective terms involving $\mathcal{L}_{B}$ and $\mathcal{L}_{S}$ in (3.14) need to be stabilized. For this purpose upwind differences for $V_{y}$ were utilized. That is, $V_{y}$ in $\mathcal{L}_{B} V$ was discretized by first-order backward differences, while $V_{y}$ in $\mathcal{L}_{S} V$ was approximated by forward differences. The same discretization was used to determine the active sets in (3.15). 
The boundary conditions (3.2) for the Newton step (3.14) read

$$
V^{n}(-1 / \gamma)=V^{n}(1 / \gamma)= \begin{cases}0, & \text { if } 0<\alpha<1 \\ -\infty, & \text { if } \alpha<0\end{cases}
$$

and thus they require special care if $\alpha<0$. Regardless of the sign of $\alpha$ we shall exploit the fact that the buy and sell trading regions extend to the boundaries $-1 / \gamma$ and $1 / \gamma$, respectively. This follows from the boundary conditions (2.14) with arguments as used to derive the corresponding Corollaries 8.7/8.8 in ?. That is, the solution of the continuous problem satisfies $\mathcal{L}_{B} V(t, y)=0$ for $y$ near $-1 / \gamma$ at all times $t \in[0, T)$, i.e.,

$$
V(t, y)=c_{B}(t)(1+\gamma y)^{\alpha}
$$

holds with an unknown integration constant $c_{B}(t) \neq 0$.

Suppose that $a \in(-1 / \gamma, \sup B(t))$ is a given number in the buy region. From $\mathcal{L}_{B} V(t, a)=0$ we infer

$$
(1+\gamma a) V_{y}(t, a)-\alpha \gamma V(t, a)=0 .
$$

Similarly, we obtain for $b \in(\inf S(t), 1 / \gamma)$ the condition

$$
-(1+\gamma b) V_{y}(t, b)-\alpha \gamma V(t, b)=0 .
$$

Thus using the Robin boundary conditions (3.17) allows us to solve the Newton step (3.14) on the subdomain $(a, b) \subset(-1 / \gamma, 1 / \gamma)$ only. We refer to this as the reduced domain technique and it leads to a significant reduction of the size of the computational domain. The unknown constants $c_{B}(t)$ and similarly $c_{S}(t)$ can be computed a posteriori from

$$
c_{B}(t)=V(t, a)(1+\gamma a)^{-\alpha}, \quad c_{S}(t)=V(t, b)(1-\gamma b)^{-\alpha}
$$

The solution $V(t, \cdot)$ can thus be recovered outside $(a, b)$. Note that this procedure can be used regardless of the sign of $\alpha$.

For convenience, we summarize the semi-smooth Newton time-stepping method as Algorithm 3.1.

3.5. Unregularized Active Set Method. For comparison, we also implemented an unregularized version of the semi-smooth Newton iteration. It is based on the following reformulation of (3.1) as a complementarity problem:

$$
\begin{aligned}
& V_{t}+\mathcal{L} V+\lambda_{B}+\lambda_{S}=0 \\
& \lambda_{B} \geq 0, \quad \mathcal{L}_{B} V \leq 0, \quad \lambda_{B} \mathcal{L}_{B} V=0 \\
& \lambda_{S} \geq 0, \quad \mathcal{L}_{S} V \leq 0, \quad \lambda_{S} \mathcal{L}_{S} V=0
\end{aligned}
$$

with boundary and terminal conditions (3.2)-(3.3), compare (3.10) in the discussion of the beginning of Section 3.1. Using the $\max \{0, \cdot\}$ complementarity function, (3.19) can be equivalently expressed as

$$
\begin{aligned}
& V_{t}+\mathcal{L} V+\lambda_{B}+\lambda_{S}=0 \\
& \lambda_{B}=\max \left\{0, \lambda_{B}+\varrho \mathcal{L}_{B} V\right\}, \quad \lambda_{S}=\max \left\{0, \lambda_{S}+\varrho \mathcal{L}_{S} V\right\}
\end{aligned}
$$

for any $\varrho>0$, as was explained for the introductory example in (3.11). This leads to the following semidiscrete formulation of (3.1)

$$
\begin{aligned}
& \frac{V^{n+1}-V^{n}}{\tau}+\mathcal{L}\left(\theta V^{n}+(1-\theta) V^{n+1}\right)+\lambda_{B}+\lambda_{S}=0 \\
& \lambda_{B}=\max \left\{0, \lambda_{B}+\varrho \mathcal{L}_{B} V^{n}\right\}, \quad \lambda_{S}=\max \left\{0, \lambda_{S}+\varrho \mathcal{L}_{S} V^{n}\right\}
\end{aligned}
$$




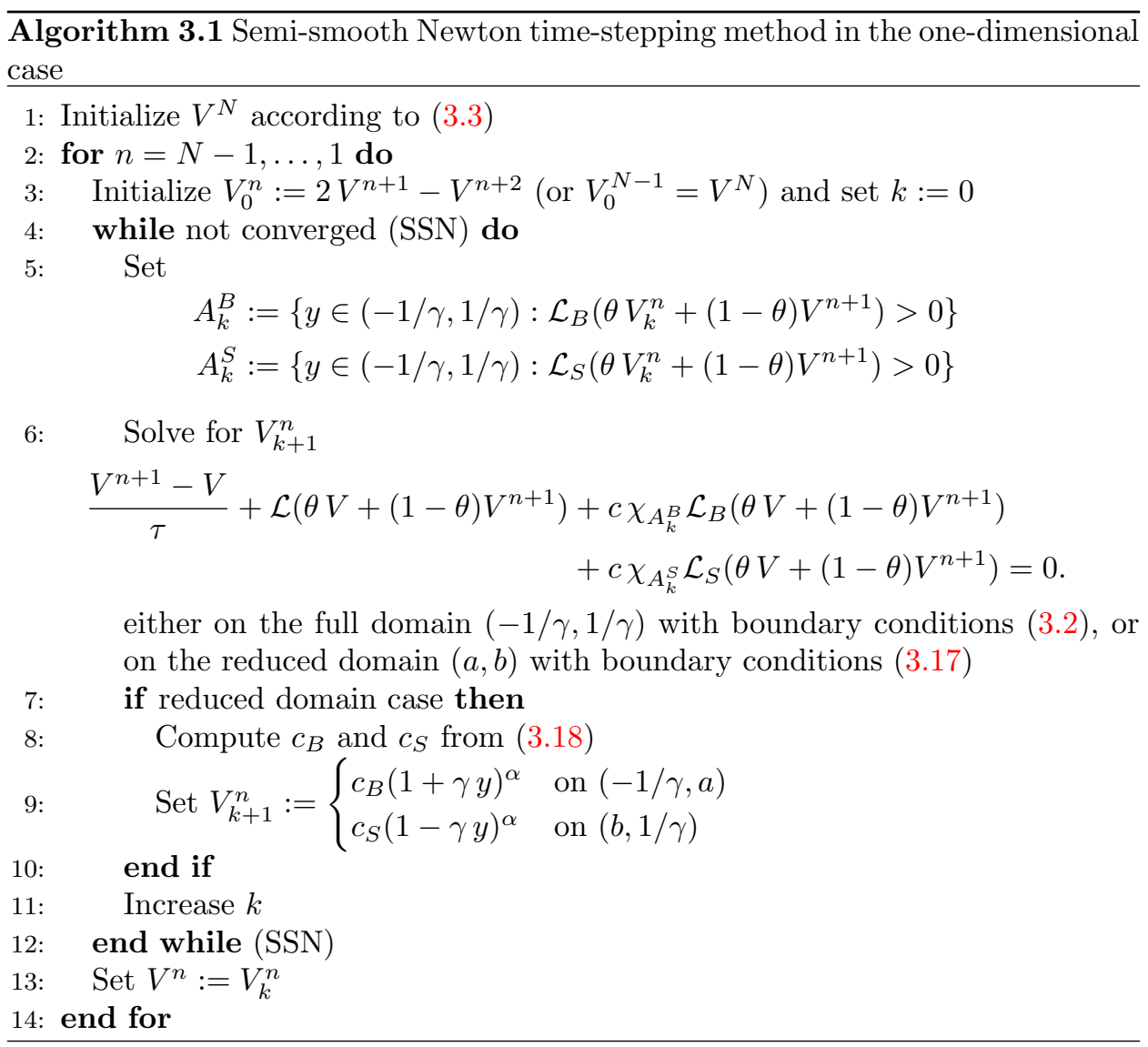

on $(0, T) \times(-1 / \gamma, 1 / \gamma)$. We suppose again that the active sets

$$
\begin{aligned}
& A^{B}=\left\{y \in(-1 / \gamma, 1 / \gamma): \lambda_{B}+\varrho \mathcal{L}_{B} V^{n}>0\right\} \\
& A^{S}=\left\{y \in(-1 / \gamma, 1 / \gamma): \lambda_{S}+\varrho \mathcal{L}_{S} V^{n}>0\right\}
\end{aligned}
$$

are intervals of the form $(-1 / \gamma, a)$ and $(b, 1 / \gamma)$. As in (3.17), we impose Robin boundary conditions at $y=a$ and $y=b$ and solve

$$
\frac{V^{n+1}-V^{n}}{\tau}+\mathcal{L}\left(\theta V^{n}+(1-\theta) V^{n+1}\right)=0 \quad \text { on }(a, b) .
$$

The equations in (3.21) are coupled through the active sets. Their iterative solution by Newton's method leads to Algorithm 3.2. Note that the interval structure of the active sets is enforced in step 8 . The initialization of $\lambda_{B, 0}^{N-1}$ and $\lambda_{S, 0}^{N-1}$ in step 5 is motivated by the update formula for $\lambda_{B}$ and $\lambda_{S}$ as applied in step 12, under the best available guess $V^{N-1}=V^{N}$.

3.6. Numerical Results. For all computations, a uniform time grid with 200 points with implicit Euler time-stepping $(\theta=1)$ was used. In order to resolve the trading bounds accurately, a refined grid with 4000 grid points in $[\hat{\eta}-1, \hat{\eta}+1]$ around the Merton fraction and a coarse grid with 100 points each away from it on $[-1 / \gamma, \hat{\eta}-1]$ and $[\hat{\eta}+1,1 / \gamma]$ was used. For all examples, the Robin-type boundary conditions (3.17) were employed on the reduced domain $(a, b)=\left(-\frac{1}{10 \gamma}, \frac{1}{10 \gamma}\right)$.

The iteration for a given time level $n$ (see steps 4-12 in Algorithm 3.1) was terminated as soon as one of the following criteria was satisfied:

(1) the active sets coincided: $A_{k+1}^{B}=A_{k}^{B}$ and $A_{k+1}^{S}=A_{k}^{S}$ 
$\overline{\text { Algorithm 3.2 Unregularized semi-smooth Newton time-stepping method in the }}$ one-dimensional case

1: Initialize $V^{N}$ according to (3.3)

2: Set

$$
\begin{aligned}
& a:=\min \left\{y \in(-1 / \gamma, 1 / \gamma): \mathcal{L} V^{N} \geq 0\right\} \\
& b:=\max \left\{y \in(-1 / \gamma, 1 / \gamma): \mathcal{L} V^{N} \geq 0\right\}
\end{aligned}
$$

3: for $n=N-1, \ldots, 1$ do

4: Initialize $V_{0}^{n}:=2 V^{n+1}-V^{n+2}\left(\right.$ or $\left.V_{0}^{N-1}=V^{N}\right)$ and set $k:=0$

5: $\quad$ Initialize $\lambda_{B, 0}^{n}:=2 \lambda_{B}^{n+1}-\lambda_{B}^{n+2}$ and $\lambda_{S, 0}^{n}:=2 \lambda_{S}^{n+1}-\lambda_{S}^{n+2}$ or

$$
\begin{array}{ll}
\lambda_{B, 0}^{N-1}:=-\mathcal{L} V^{N} & \text { on }(-1 / \gamma, a) \\
\lambda_{S, 0}^{N-1}:=-\mathcal{L} V^{N} & \text { on }(b, 1 / \gamma) \\
\lambda_{B, 0}^{N-1}:=\lambda_{S, 0}^{N-1}:=0 & \text { elsewhere }
\end{array}
$$

6: $\quad$ while not converged $(\mathrm{SSN})$ do

7: $\quad$ Set

$$
\begin{aligned}
& A_{k}^{B}:=\left\{y \in(-1 / \gamma, 1 / \gamma): \lambda_{B, k}^{n}+\varrho \mathcal{L}_{B} V_{k}^{n}>0\right\} \\
& A_{k}^{S}:=\left\{y \in(-1 / \gamma, 1 / \gamma): \lambda_{S, k}^{n}+\varrho \mathcal{L}_{S} V_{k}^{n}>0\right\}
\end{aligned}
$$

8: $\quad$ Set

$$
a:=\max A_{k}^{B} \quad \text { and } \quad b:=\min A_{k}^{S}
$$

9: $\quad$ Solve for $V_{k+1}^{n}$

$$
\frac{V^{n+1}-V}{\tau}+\mathcal{L}\left(\theta V+(1-\theta) V^{n+1}\right)=0
$$

on $(a, b)$ with boundary conditions $(3.17)$

10: $\quad$ Compute $c_{B}$ and $c_{S}$ from (3.18)

11: Set $V_{k+1}^{n}:= \begin{cases}c_{B}(1+\gamma y)^{\alpha} & \text { on }(-1 / \gamma, a) \\ c_{S}(1-\gamma y)^{\alpha} & \text { on }(b, 1 / \gamma)\end{cases}$

12: $\quad$ Set

$\lambda_{B, k+1}^{n}:=-\frac{V^{n+1}-V_{k+1}^{n}}{\tau}-\mathcal{L}\left(\theta V_{k+1}^{n}+(1-\theta) V^{n+1}\right) \quad$ on $(-1 / \gamma, a)$

$\lambda_{S, k+1}^{n}:=-\frac{V^{n+1}-V_{k+1}^{n}}{\tau}-\mathcal{L}\left(\theta V_{k+1}^{n}-(1-\theta) V^{n+1}\right) \quad$ on $(b, 1 / \gamma)$

and $\lambda_{B, k+1}^{n}=\lambda_{S, k+1}^{n}=0$ elsewhere

13: $\quad$ Increase $k$

14: end while $(\mathrm{SSN})$

15: $\quad$ Set $V^{n}:=V_{k}^{n}, \lambda_{B}^{n}:=\lambda_{B, k}^{n}$ and $\lambda_{S}^{n}:=\lambda_{S, k}^{n}$

16: end for

(2) the time step residual

$\left\|\max \left\{\frac{V^{n+1}-V_{k+1}^{n}}{\tau}-\mathcal{L}\left(\theta V_{k+1}^{n}+(1-\theta) V^{n+1}\right), \mathcal{L}_{B} V_{k+1}^{n}, \mathcal{L}_{S} V_{k+1}^{n}\right\}\right\|_{L^{\infty}(\mathcal{S})}$

after step 10 was below $10^{-7}$,

(3) the terms determining the change of active sets

$$
\begin{aligned}
j_{B} & :=\mathcal{L}_{B}\left(\theta V_{k+1}^{n}+(1-\theta) V^{n+1}\right) \\
j_{S} & :=\mathcal{L}_{S}\left(\theta V_{k+1}^{n}+(1-\theta) V^{n+1}\right)
\end{aligned}
$$


after step 10 satisfied

$$
\begin{array}{ll}
\left|j_{B}\right|<10^{-6} & \text { on } A_{k}^{B} \backslash A_{k+1}^{B} \text { and on } A_{k+1}^{B} \backslash\left(A_{k}^{B} \cup A_{k}^{S}\right) \\
\left|j_{S}\right|<10^{-6} & \text { on } A_{k}^{S} \backslash A_{k+1}^{S} \text { and on } A_{k+1}^{S} \backslash\left(A_{k}^{B} \cup A_{k}^{S}\right) .
\end{array}
$$

In all examples, criteria (1) or (3) were always satisfied first.

Example 3.1. In this example we consider the following problem data

$$
\begin{aligned}
& \alpha=0.1 \quad \text { utility exponent } \quad \gamma=1.0 \% \quad \text { trading costs } \\
& \mu=9.6 \% \quad \text { stock trend } \quad \sigma=0.4 \quad \text { stock volatility } \\
& r=0.0 \% \text { interest rate }
\end{aligned}
$$

on the time interval $(0,1)$. The Merton fraction for this example is $\hat{\eta}=2 / 3$, and we consider the case with liquidation costs.

We report on the performance of Algorithm 3.1 in a MATLAB implementation with various choices of the regularization parameter $c$, see Table 3.1. The plot of the trading region boundaries is shown in Figure 3.1. The run-time was never more than 7 seconds on a contemporary PC.

The area between the black and the red curve is the no-trading region $N T$. If at time $t$ a fraction $y \in N T(t)$ of our wealth is invested in the stocks, we would not trade. Below from the black curve we have the buy region $B$, above from the red curve the sell region $S$. Starting with a risky fraction in $N T$ we actually only touch the boundary of $B$ or $S$ as described in the introduction. But starting at $t \in[0, T)$ with a risky fraction in the interior of $B(t)$ or $S(t)$, it would be optimal to buy or sell, respectively, in such a way that after trading the new risky fraction lies on the boundary of $N T(t)$ and afterwards to continue as above.

The financial interpretation of the shape of the regions in Figure 3.1 is as follows: The Merton fraction $\hat{\eta}=2 / 3>0$ indicates that it is optimal to invest in the stocks. It is desirable to stay close to $\hat{\eta}$. From Figure 3.1 we learn that for positions above $\hat{\eta}$ (dashed line) it becomes more and more attractive to sell stocks (red curve) to get closer to $\hat{\eta}$, since we have to sell all stocks at terminal time anyway. On the other hand, it is always better to be $100 \%$ invested in the bond $(y=0)$, than to have a short position in the stocks $(y<0)$. Due to the liquidation costs at terminal time we would liquidate a short position in the stocks immediately (positive black curve). If the expected gains from the stock investment are higher than the costs, we buy stocks, as is the case for $t<0.78$ (black curve strictly positive). The influence of these small realistic costs of $\gamma=1 \%$ can be seen very well from the solid blue curve which shows that it would be better to start at $t=0.9$ with a position zero in the stocks than at the Merton fraction $\hat{\eta}$, which is optimal without costs. In the short remaining time the liquidation costs are higher than the expected proceeds from the stock investment.

In Figure 3.2, the three terms in

$$
\max \left\{\frac{V^{n+1}-V^{n}}{\tau}-\mathcal{L}\left(\theta V^{n}+(1-\theta) V^{n+1}\right), \quad \mathcal{L}_{B} V^{n}, \quad \mathcal{L}_{S} V^{n}\right\}=0
$$

are depicted in the vicinity of the trading bounds. Note that the angle of intersection between these curves is small. Hence the sensitivity of the boundaries of the trading regions with respect to the penalty parameter $c$ must be checked. To this end, the values $\triangle b$ and $\triangle s$ are included in Table 3.1. As can be seen, the boundaries converge as $c$ increases. Moreover, the table gives a clear indication that the residual error is linked to regularization.

For the sake of comparison, we also applied the unregularized Algorithm 3.2 for this and the following example. The reduced computational domain was chosen to 


\begin{tabular}{lllll}
\hline$c$ & iter & $\triangle b$ & $\triangle s$ & residual \\
\hline 1.00E0 & 319 & - & - & $4.00 \mathrm{E}-1$ \\
$1.00 \mathrm{E} 1$ & 459 & 0.0040 & 0.0040 & $1.97 \mathrm{E}-1$ \\
$1.00 \mathrm{E} 2$ & 524 & 0.0005 & 0.0005 & $4.15 \mathrm{E}-2$ \\
$1.00 \mathrm{E} 3$ & 539 & 0.0005 & 0.0005 & $6.61 \mathrm{E}-3$ \\
$1.00 \mathrm{E} 4$ & 543 & 0.0000 & 0.0005 & $8.15 \mathrm{E}-4$ \\
$1.00 \mathrm{E} 5$ & 550 & 0.0005 & 0.0005 & $8.76 \mathrm{E}-5$ \\
\hline \hline
\end{tabular}

TABle 3.1. Performance of Algorithm 3.1 for Example 3.1 with implicit Euler time-stepping $(\theta=1)$ for various values of the regularization parameter $c$. 'iter' refers to the total number of iterations, accumulated over all 200 time steps, and 'residual' denotes the expression in (3.22) at time $t=0$. Moreover, ' $\triangle b$ ' denotes the shift of the computed buy trading boundary at time $t=0$, compared to the previous value of $c$. It is a multiple of the local mesh size 0.0005 . ' $\triangle s^{\prime}$ is the same for the sell trading boundary.

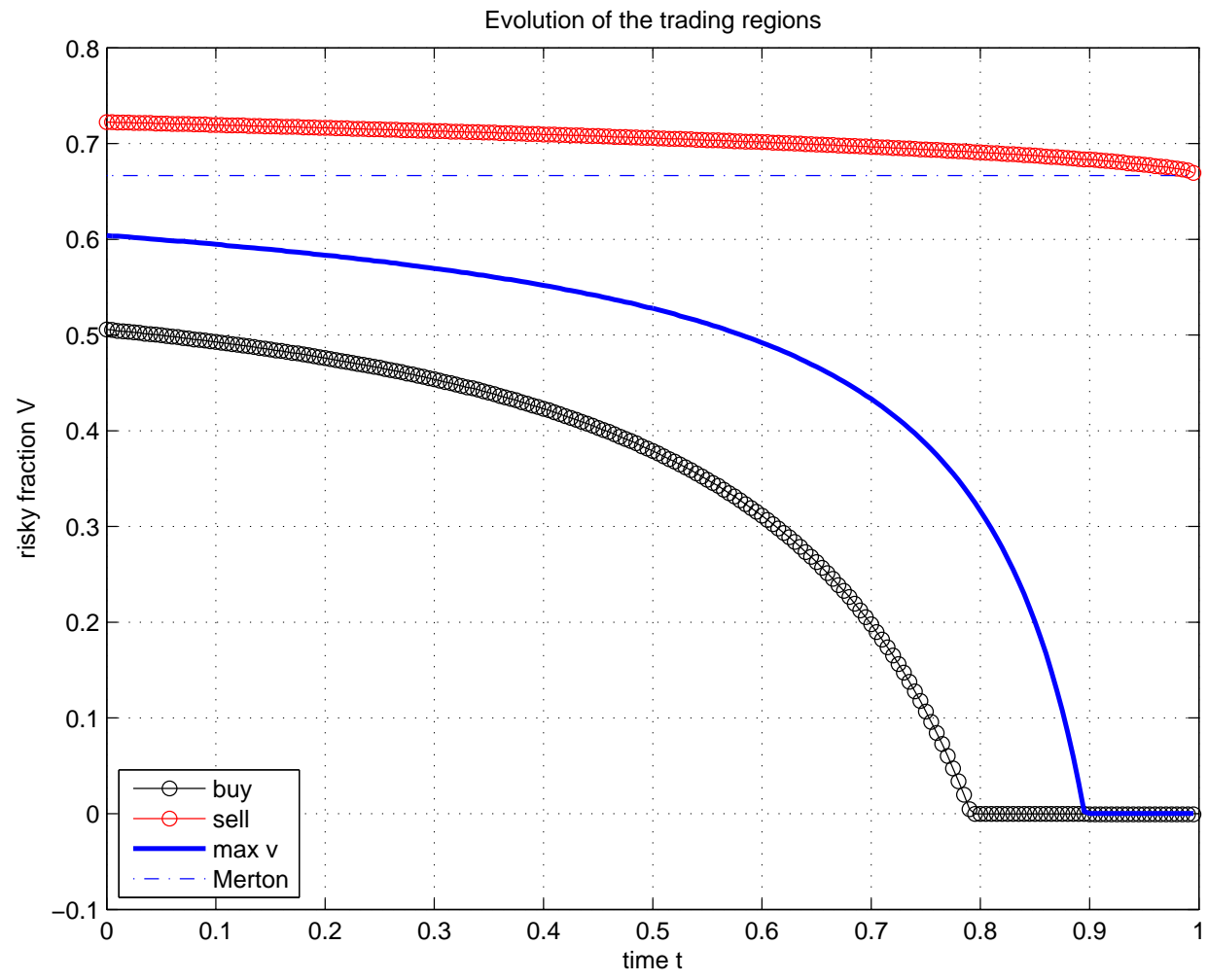

FiguRE 3.1. Boundaries of the trading regions in Example 3.1 (red and black). The figure also shows the Merton fraction (dashdotted) and the risky fraction where $V$ assumes its maximum at any given time (solid line).

be $(a, b)=\left(-\frac{1}{3 \gamma}, \frac{1}{3 \gamma}\right)$ in these cases. With the same stopping criteria in place and $\varrho=10^{1}$, the residual error at $t=0$ was found to be $3.47 \cdot 10^{-5}$ and $4.60 \cdot 10^{-6}$, respectively. It is thus comparable than the residuals obtained by the regularized 


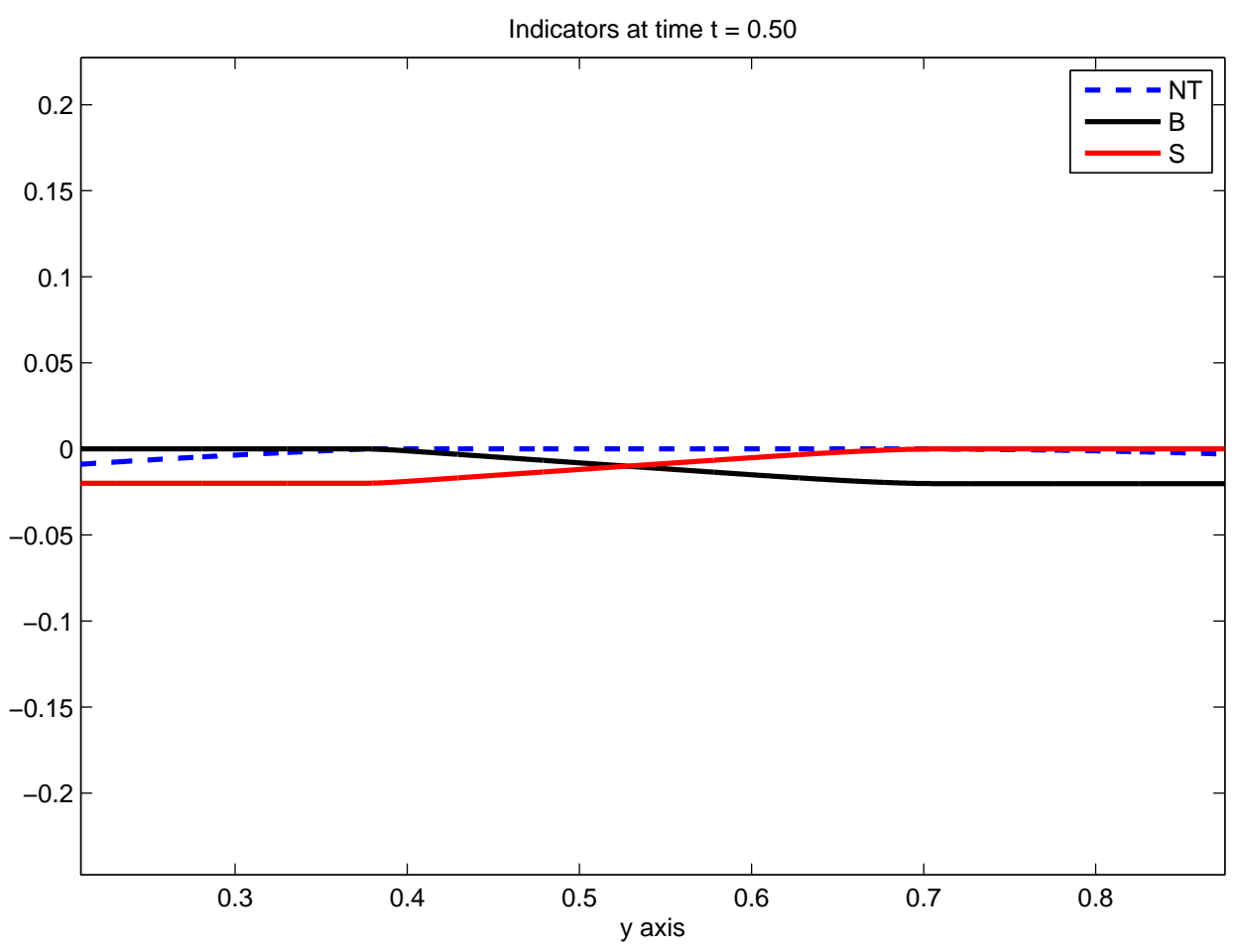

FiguRE 3.2. Plot of the three terms in (3.24) at $t=0.5$ near the trading boundaries $b(t) \approx 0.38$ and $s(t) \approx 0.71$.

algorithm (Algorithm 3.1) for appropriate values of c, compare Tables 3.1 and 3.2. A total number of 645 and 526 iterations were needed for all 200 time steps.

We also remark that the use of appropriate initialization of the Lagrange multipliers given in Step 5 of the Algorithm 3.2 as well as Step 8, which enforces the interval structure of the no-trading region, are essential.

Example 3.2. Here the previous problem is modified by choosing the more riskaverse parameter

$$
\alpha=-1.0 \quad \text { utility exponent. }
$$

The Merton fraction is now $\hat{\eta}=0.3$. In Figure 3.3 we see that we have a similar interpretation as in Figure 3.1 w.r.t. to the Merton fraction which is now smaller due to the more risk averse utility function.

We report again on the performance of Algorithm 3.1 for various choices of the regularization parameter $c$, see Table 3.2. As discussed before, in view of $\alpha<0$ boundary conditions (3.17) are employed. As the reduced domain of computation, we used again $(a, b)=\left(-\frac{1}{10 \gamma}, \frac{1}{10 \gamma}\right)$.

Example 3.3. In a third example, we modified Example 3.1 by choosing

$$
\mu=-10.0 \% \text { stock trend. }
$$

The Merton fraction is $\hat{\eta}=-0.6944$ in this example.

In Figure 3.4 we see that we have qualitatively a mirrored plot compared to Figure 3.1 of Example 3.1. This is due to the fact that we now have a negative trend $\mu$ for which it can be shown that holding stocks $(y>0)$ can never be optimal. It is preferable to be short in the stocks $(y<0)$ which allows us to profit from decreasing stock prices. Liquidation now means that we have to buy stocks at terminal 
PRIMAL-DUAL METHODS FOR COMPUTATION OF TRADING REGIONS

\begin{tabular}{lllll}
\hline$c$ & iter & $c_{B}$ & $c_{S}$ & residual \\
\hline $1.00 \mathrm{E} 2$ & 457 & -2.4561 & -1.8063 & $3.21 \mathrm{E}-1$ \\
$1.00 \mathrm{E} 3$ & 459 & -1.0835 & -1.0474 & $1.82 \mathrm{E}-2$ \\
$1.00 \mathrm{E} 4$ & 460 & -1.0086 & -0.9955 & $2.02 \mathrm{E}-3$ \\
$1.00 \mathrm{E} 5$ & 462 & -1.0013 & -0.9905 & $2.18 \mathrm{E}-4$ \\
$1.00 \mathrm{E} 6$ & 460 & -1.0006 & -0.9899 & $9.02 \mathrm{E}-5$ \\
\hline \hline
\end{tabular}

TABle 3.2. Performance of Algorithm 3.1 for Example 3.2. See Table 3.1 for a legend. In addition, $c_{B}$ and $c_{S}$ denote the values of these constants, see (3.18), at time $t=0$.

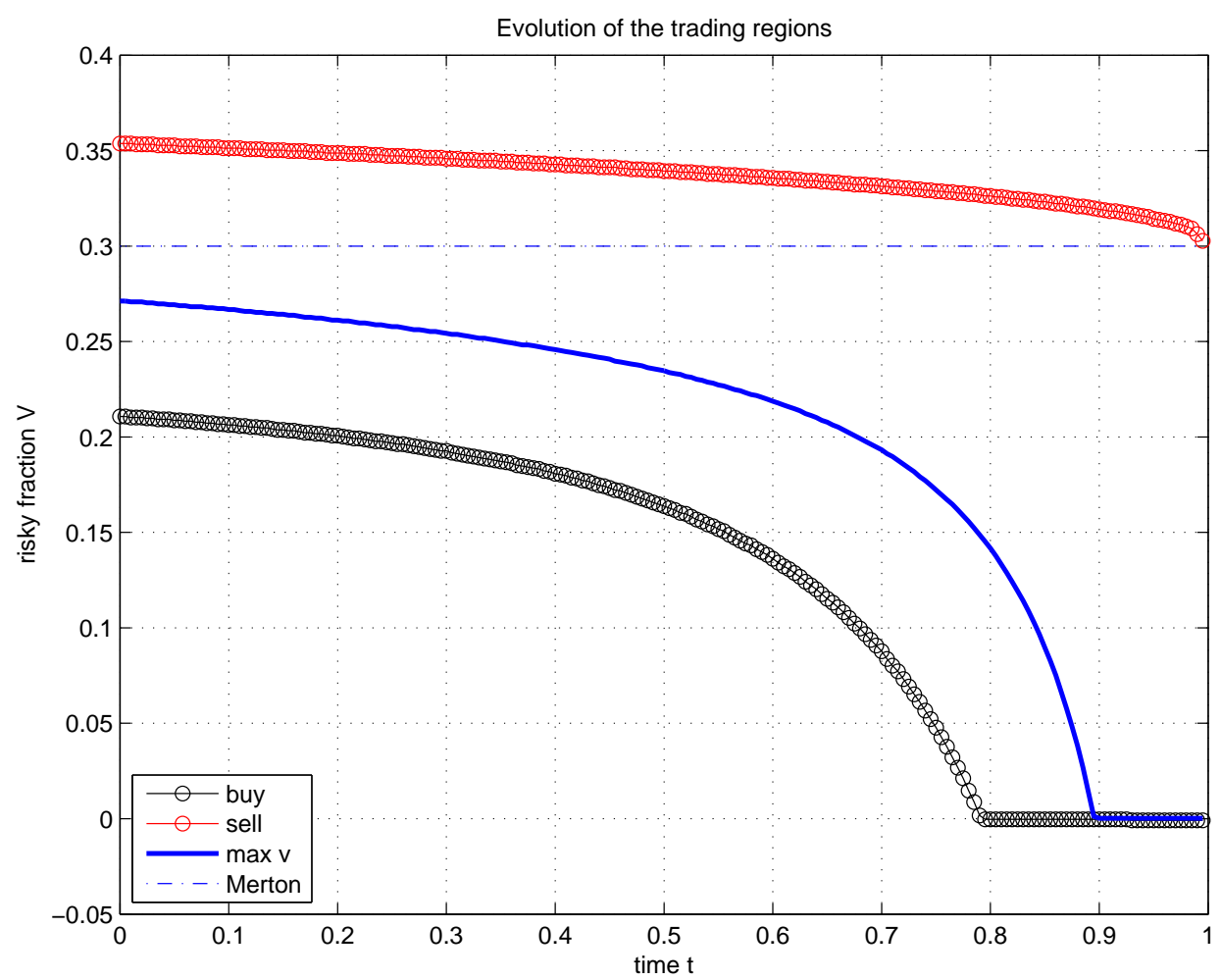

FiguRE 3.3. Boundaries of the trading regions in Example 3.2 (red and black). See Figure 3.1 for a legend.

time to close the short position in the stocks, and the same arguments as for the interpretation of Figure 3.1 apply.

We report once again on the performance of Algorithm 3.1 for various choices of the regularization parameter $c$, see Table 3.3.

\section{The Case of Two Risky Assets}

In the case of two risky assets $n=2$, the solution of equation (2.15) becomes significantly more involved. We recall that the solvency region

$$
\mathcal{S}=\left\{y \in \mathbb{R}^{2}:\|y\|_{1}<1 / \gamma\right\}
$$




\begin{tabular}{lcc}
\hline$c$ & iter & residual \\
\hline $1.00 \mathrm{E} 0$ & 259 & $5.69 \mathrm{E}-1$ \\
$1.00 \mathrm{E} 1$ & 367 & $2.59 \mathrm{E}-1$ \\
$1.00 \mathrm{E} 2$ & 436 & $4.86 \mathrm{E}-2$ \\
$1.00 \mathrm{E} 3$ & 444 & $6.83 \mathrm{E}-3$ \\
$1.00 \mathrm{E} 4$ & 445 & $8.07 \mathrm{E}-4$ \\
$1.00 \mathrm{E} 5$ & 446 & $8.46 \mathrm{E}-5$ \\
\hline \hline
\end{tabular}

TABle 3.3. Performance of Algorithm 3.1 for Example 3.3. See Table 3.1 for a legend.

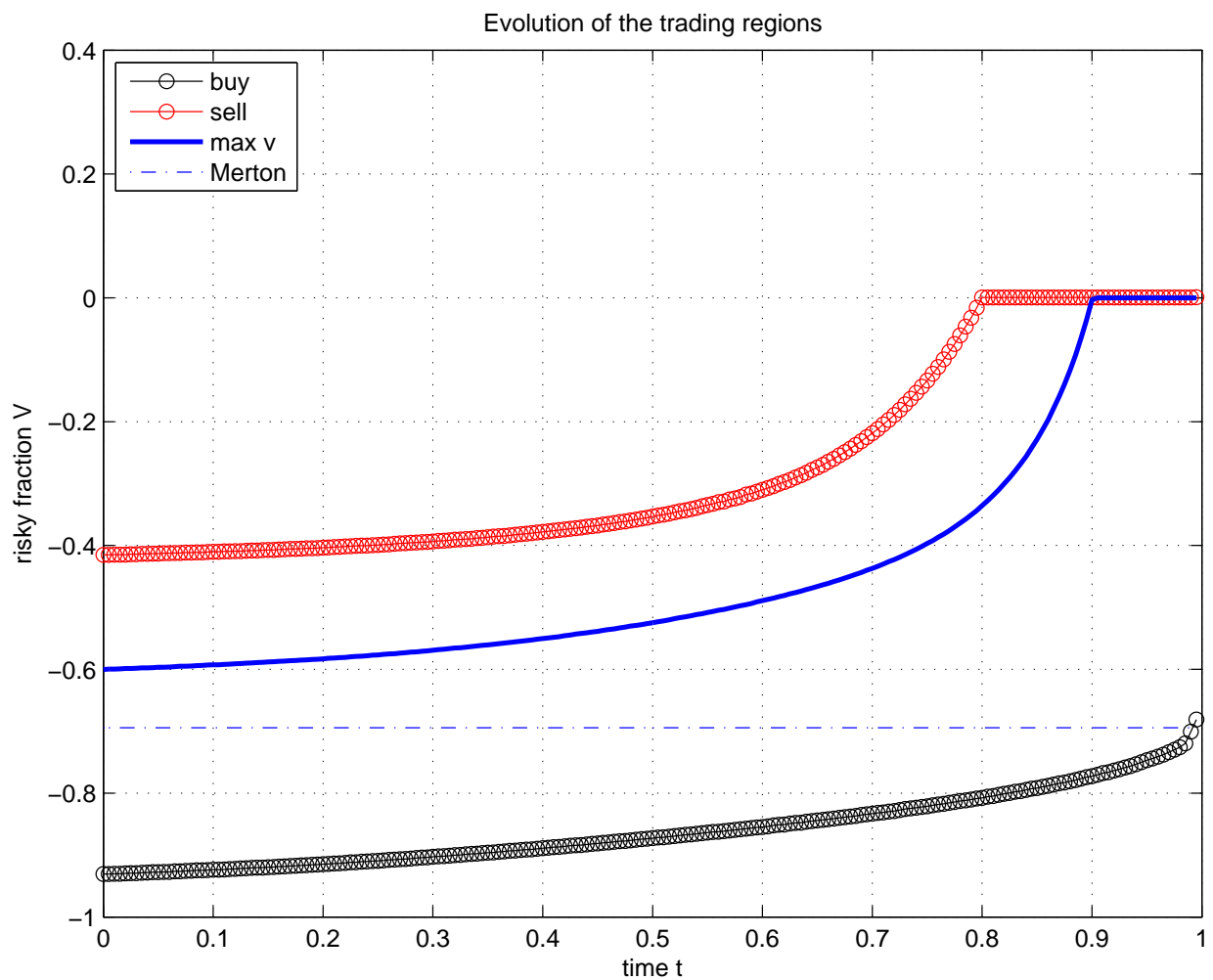

Figure 3.4. Boundaries of the trading regions in Example 3.3 (red and black). See Figure 3.1 for a legend.

is a diamond-shaped subset of $\mathbb{R}^{2}$. Parallel to the regularized formulation in the $1 \mathrm{D}$ case, see $(3.5)$, we consider

$$
V_{t}+\mathcal{L} V+c \sum_{j=1}^{2} \max \left\{0, \mathcal{L}_{B_{j}} V\right\}+c \sum_{j=1}^{2} \max \left\{0, \mathcal{L}_{S_{j}} V\right\}=0
$$

on $[0, T) \times \mathcal{S}$ with regularization parameter $c>0$ and subject to the boundary conditions (2.14). The terminal conditions for $V$ on $\mathcal{S}$ are given by

$$
\begin{aligned}
& V(T, y)=\frac{1}{\alpha} \\
& \text { without liquidation costs } \\
& V(T, y)=\frac{1}{\alpha}\left(1-\gamma\|y\|_{1}\right)^{\alpha} \\
& \text { with liquidation costs. }
\end{aligned}
$$


Similar to the 1D case (3.13) we discretize (4.2) in time to obtain

$$
\begin{array}{r}
\frac{V^{n+1}-V^{n}}{\tau}+\mathcal{L}\left(\theta V^{n}+(1-\theta) V^{n+1}\right)+c \sum_{j=1}^{2} \max \left\{0, \mathcal{L}_{B_{j}}\left(\theta V^{n}+(1-\theta) V^{n+1}\right)\right\} \\
+c \sum_{j=1}^{2} \max \left\{0, \mathcal{L}_{S_{j}}\left(\theta V^{n}+(1-\theta) V^{n+1}\right)\right\}=0 .
\end{array}
$$

We then solve (4.4) by a semi-smooth Newton iteration as in (3.14)-(3.15).

As will be described in the next subsection, the practical realization of the semismooth Newton iteration has to take into account the significant influence of convection induced by the buy and sell operators on the respective active sets. Stabilization is necessary even for small values of the regularization parameter $c$. In order to balance the influence of regularization error (which is small for large $c$ ) and of the magnitude of convection (which is small for small $c$ ), we found it favorable to work with moderate values for $c$. To reduce the influence of the incurred remaining regularization error, we embed the semi-smooth Newton iteration for each time step into an Augmented Lagrangian (ALM) loop. That is, (4.4) is replaced by

$$
\begin{aligned}
\frac{V^{n+1}-V^{n}}{\tau}+\mathcal{L}\left(\theta V^{n}+\right. & \left.(1-\theta) V^{n+1}\right)+\sum_{j=1}^{2} \max \left\{0, \lambda_{B_{j}}+c \mathcal{L}_{B_{j}}\left(\theta V^{n}+(1-\theta) V^{n+1}\right)\right\} \\
& +\sum_{j=1}^{2} \max \left\{0, \lambda_{S_{j}}+c \mathcal{L}_{S_{j}}\left(\theta V^{n}+(1-\theta) V^{n+1}\right)\right\}=0
\end{aligned}
$$

During one semi-smooth Newton loop for (4.5), the Lagrange multiplier estimates $\lambda_{B_{j}}$ and $\lambda_{S_{j}}$ remain unchanged. Once the Newton iteration for $V^{n}$ terminates, $\lambda_{B_{j}}$ and $\lambda_{S_{j}}$ are updated according to

$$
\begin{aligned}
\lambda_{B_{j}} & :=\max \left\{0, \lambda_{B_{j}}+c \mathcal{L}_{B_{j}}\left(\theta V^{n}+(1-\theta) V^{n+1}\right)\right\} \\
\lambda_{S_{j}} & :=\max \left\{0, \lambda_{S_{j}}+c \mathcal{L}_{S_{j}}\left(\theta V^{n}+(1-\theta) V^{n+1}\right)\right\}
\end{aligned}
$$

for $j=1,2$. At the beginning of each time step, all $\lambda_{B_{j}}$ and $\lambda_{S_{j}}$ are initialized by constant extrapolation from the previous time step. Upon termination of the Augmented Lagrangian loop in any given time step, complementarity systems similar to (3.21) for the $1 \mathrm{D}$ case are satisfied.

The complete Augmented Lagrangian semi-smooth Newton algorithm is given as Algorithm 4.1.

4.1. Discretization in Space and Computational Domain. The spatial discretization of the value function $V$ is based upon linear continuous finite elements. This choice offers more flexibility, e.g., with respect to local grid refinement, than does the finite difference approach. In order to assemble the weak form of $\mathcal{L}$, see (2.17a), we convert the second-order contributions in $\mathcal{L}$ into divergence form using

$$
C_{0} \circledast V_{y y}=\operatorname{div}\left(C_{0} \nabla V\right)-\sum_{i=1}^{n} \frac{\partial C_{0}}{\partial y_{j}} \frac{\partial}{\partial y_{j}} V,
$$

where $\circledast$ denotes the Hadamard product of matrices, i.e., $(A \circledast B)_{i, j}=A_{i, j} B_{i, j}$. The coefficient matrix is given by

$$
\left(C_{0}\right)_{k, l}=\frac{1}{2} \sum_{i, j=1}^{n} A_{i j} y_{i} y_{j}\left(\delta_{i k}-y_{k}\right)\left(\delta_{j l}-y_{l}\right),
$$


where $\delta_{i k}$ denotes the Kronecker delta symbol. This conversion into divergence form incurs an additional convection term, the last term in (4.6), which needs to be added to the connection terms already present in (2.17a).

In order to accomodate potentially highly convective contributions in (4.5), the discretization of the first-order terms is based upon an upwind triangle stabilization, as described for instance in [?, Chapter III, Section 3]. This applies to the convective terms in $\mathcal{L}$ as well as to those in the buy and sell operators $\mathcal{L}_{B_{j}}$ and $\mathcal{L}_{S_{j}}$, see (2.17).

It is well known that for the range of problem parameters of interest, the no-trading region is small and well inside the solvency region (4.1). This was also already observed in the one-dimensional case treated in Section 3. Together with the fact that our main interest lies in the no-trading region and the optimal trading structure in its neighborhood, this suggests once again a restriction of the computational domain. We choose our computational domain as the diamond-shaped region

$$
\mathcal{S}_{\text {red }}=\left\{y \in \mathbb{R}^{2}:\|y-\hat{\eta}\|_{1}<R\right\}
$$

centered around the Merton fraction, where $R>0$ is chosen problem dependent.

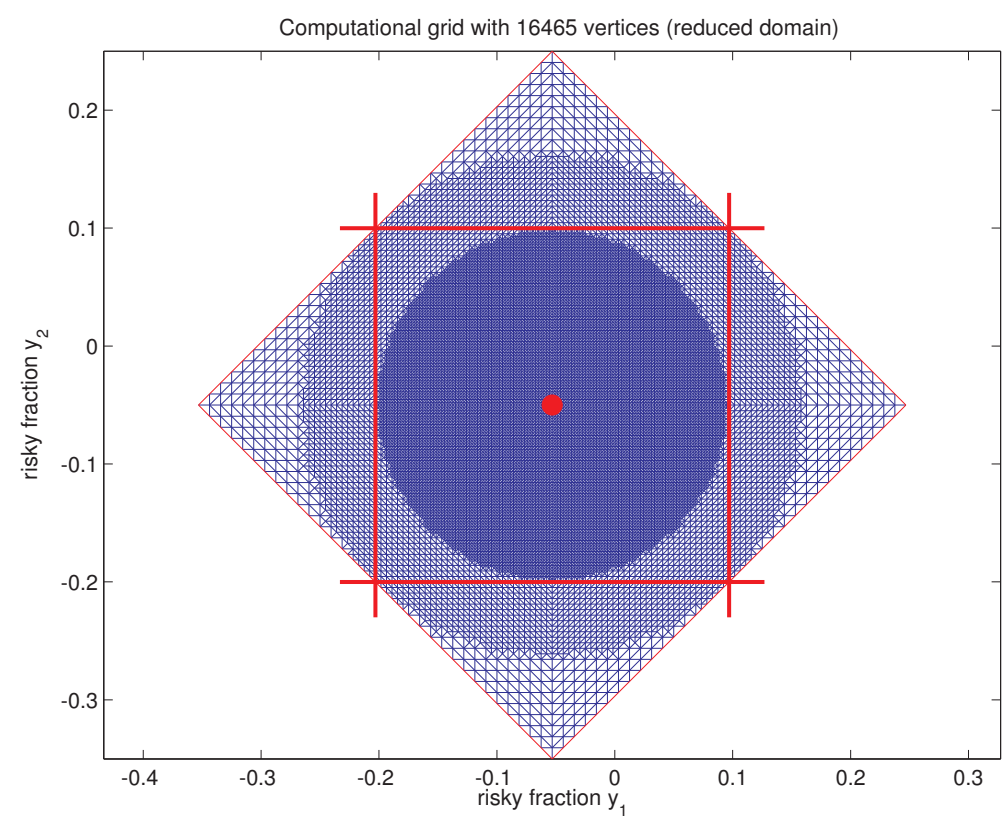

FIGURE 4.1. Example of a computational grid on the reduced domain $\mathcal{S}_{\text {red }}$ centered at the Merton fraction. The regions outside the inner square pertain to the assisted active set strategy (4.7).

The choice of boundary conditions on $\partial \mathcal{S}_{\text {red }}$ is based on the current configuration of the active sets. Since the boundary conditions are not of variational type, we adjoin them as equality constraints to the linear system (4.5) by introducing additional Lagrange multipliers. This converts (4.5) to a saddle point problem of the form

$$
\left[\begin{array}{cc}
A & B^{\top} \\
B & 0
\end{array}\right]\left(\begin{array}{l}
V \\
p
\end{array}\right)=\left(\begin{array}{l}
b \\
0
\end{array}\right)
$$

The rows of $B$ have, for instance, entries of the form

$$
\int_{\partial \mathcal{S}_{\text {red }}} \mathcal{L}_{B_{1}} \varphi_{j} \varphi_{i} \mathrm{~d} s, \quad j=1, \ldots, n_{\text {nodes }},
$$


where node $i \in A_{B_{1}} \cap N T_{2}$. At parts of the boundary which belong to two active sets, we use the sum of the two contributions from each of them.

It is known that the no-trading region is enclosed to the left and the right by buy and sell regions for the first asset, and analogously on top and below by the second asset. This knowledge is used to assist the choice of active sets in Algorithm 4.1, step 9. Indeed, in practice we use

$$
\begin{gathered}
\widetilde{A}_{k}^{B_{j}}:=A_{k}^{B_{j}} \cup\left\{y \in \mathbb{R}^{2}: y_{j}-\hat{\eta}_{j} \leq-R / 2\right\}, \quad j=1,2, \\
\widetilde{A}_{k}^{S_{j}}:=A_{k}^{S_{j}} \cup\left\{y \in \mathbb{R}^{2}: y_{j}-\hat{\eta}_{j} \geq R / 2\right\}, \quad j=1,2 .
\end{gathered}
$$

The assist strategy can be interpreted as applying boundary conditions in a penalized form in a square of side length $R$ centered at the Merton fraction which is inscribed into $\mathcal{S}_{\text {red, }}$, see Figure 4.1.

The graphical representation of the trading regions in the examples below is based on an inexpensive postprocessing step in which the active sets on every time level $n$ are determined according to the unstabilized buy and sell operators, i.e.,

$$
\begin{aligned}
& A^{B_{j}}=\left\{y \in \mathcal{S}_{\text {red }}: \mathcal{L}_{B_{j}}^{\text {unstab }} V^{n}>0\right\} \\
& A^{S_{j}}=\left\{y \in \mathcal{S}_{\text {red }}: \mathcal{L}_{S_{j}}^{\text {unstab }} V^{n}>0\right\} .
\end{aligned}
$$

4.2. Adaptive Time Stepping. Algorithm 4.1 is stated for a predetermined number $N$ of time steps of fixed length $\tau$. Computational experience shows that the changes in the trading regions can be highly varying in time, especially near the final time $T$. This suggests the use of an adaptive time stepping procedure. We gave preference to a simple heuristic procedure over classical ones. Our target is to choose the time step size $\tau_{n}$ such that

$$
d_{\mathrm{rel}}:=\frac{\left\|\nabla\left(V^{n+1}-V^{n}\right)\right\|_{L^{2}\left(\mathcal{S}_{\mathrm{red}}\right)}}{\left\|\nabla V^{n}\right\|_{L^{2}\left(\mathcal{S}_{\mathrm{red}}\right)}} \approx C .
$$

The new time step is chosen as $\min \left\{\max \left\{\tau_{n} / d_{\text {rel }}, \tau_{\min }\right\}, \tau_{\max }\right\}$ where $\tau_{\min }=10^{-4}$ and $\tau_{\max }=5 \cdot 10^{-2}$. A time step is accepted if $d_{\text {rel }} / C<1.2$ or if $\tau_{n}=10^{-4}$, and otherwise rejected. Typically, rejection only occured at the first time step when the initial $\tau$ was chosen too large.

As in the $1 \mathrm{D}$ case, we used the implicit Euler time-stepping scheme $(\theta=1)$ for all computations.

4.3. Numerical Results. The iteration for any given time level $n$ of the SSN loop was terminated as soon as one the following criteria were met:

(1) the active sets coincided,

(2) jump terms analogously defined as in (3.23) were below $10^{-9}$,

(3) the relative change between iterations in the value function

$$
\frac{\left\|V_{k+1}^{n}-V_{k}^{n}\right\|_{\left.L^{\infty}\left(\mathcal{S}_{\mathrm{red}}\right)\right)}}{\left\|V_{k+1}^{n}\right\|_{\left.L^{\infty}\left(\mathcal{S}_{\mathrm{red}}\right)\right)}}
$$

was below $10^{-12}$.

Typically, criteria (1) or (3) were satisfied first with only very few occurences of criterion (2). This clarifies the stopping in step 8 of Algorithm 4.1.

The outer ALM loop was essential in converging the trading regions especially in the first few time steps (where time $t$ is near $T$ ). We found three ALM steps to be sufficient in each case. 


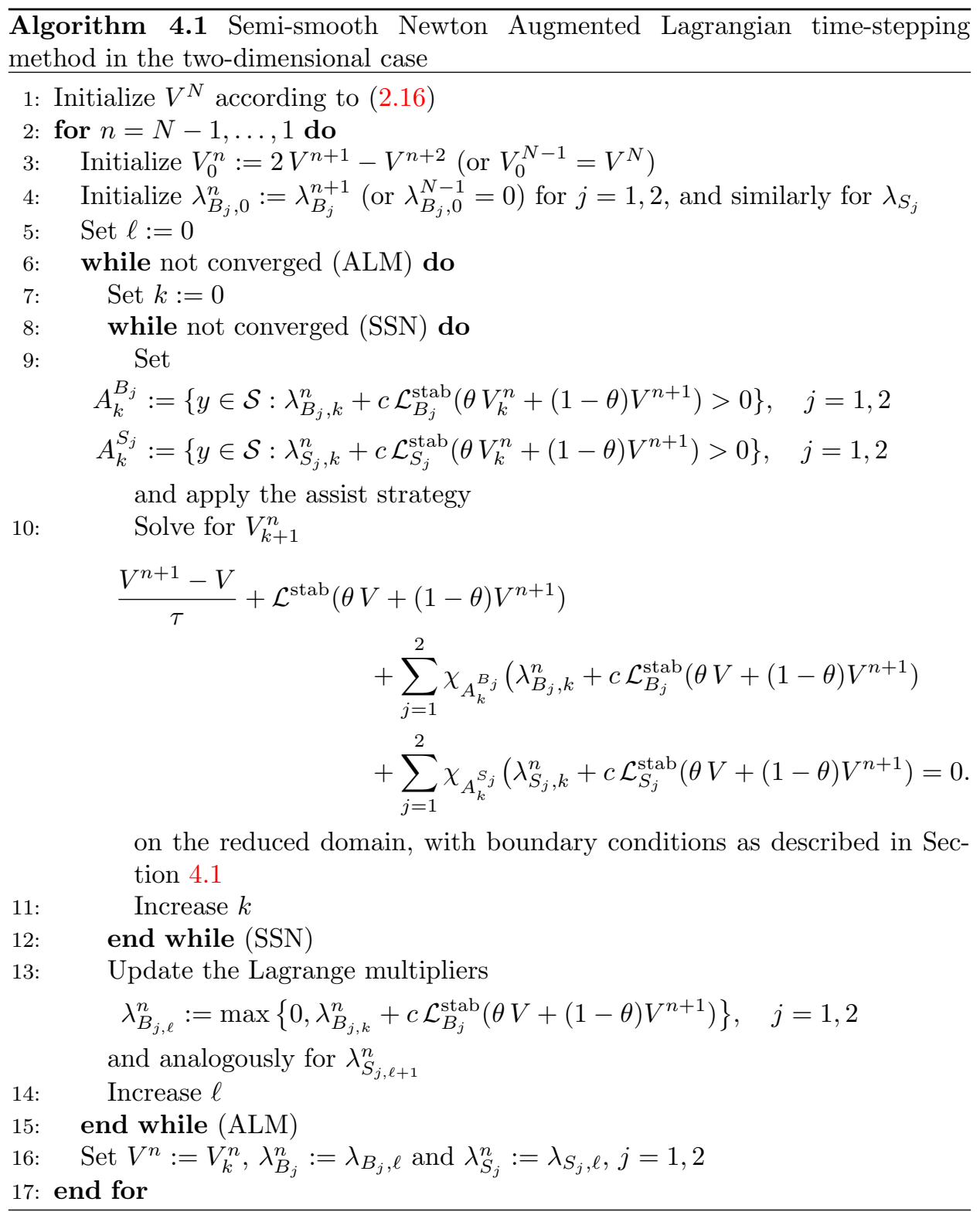

Example 4.1. In our first example, we used the following problem data:

$$
\begin{array}{llll}
\alpha=-0.5 & \text { utility exponent } & \gamma=0.5 \% & \text { trading costs } \\
\mu=\left(\begin{array}{lll}
-1.0 \% \\
-1.5 \%
\end{array}\right) & \text { stock trends } & \sigma=\left(\begin{array}{ll}
0.30 & 0.05 \\
0.05 & 0.40
\end{array}\right) & \text { stock volatilities } \\
r=0.0 \% & \text { interest rate } & &
\end{array}
$$

on the time interval $(0,1)$. The Merton fraction is $\hat{\eta}=(-0.0531,-0.0501)^{\top}$, and we consider the case with liquidation costs.

We ran Algorithm 4.1 with regularization parameter $c=10^{5}$ on a reduced domain of radius $R=0.3$. With a target relative change of $\nabla V$ in between time steps of $C=5 \cdot 10^{-3}$, see (4.9), the algorithm used 104 time steps. The evolution of time step sizes is shown in Figure 4.3. The nearly constant time steps reflect the relatively gentle motion of the trading regions (see Figure 4.2), which is due to 
the utility exponent $\alpha=-0.5$, representing a high level of relative risk aversion $1-\alpha=1.5$. Approximately 8 semi-smooth Newton steps were used on average per time step. To show that our method is not restricted to the simplex we chose
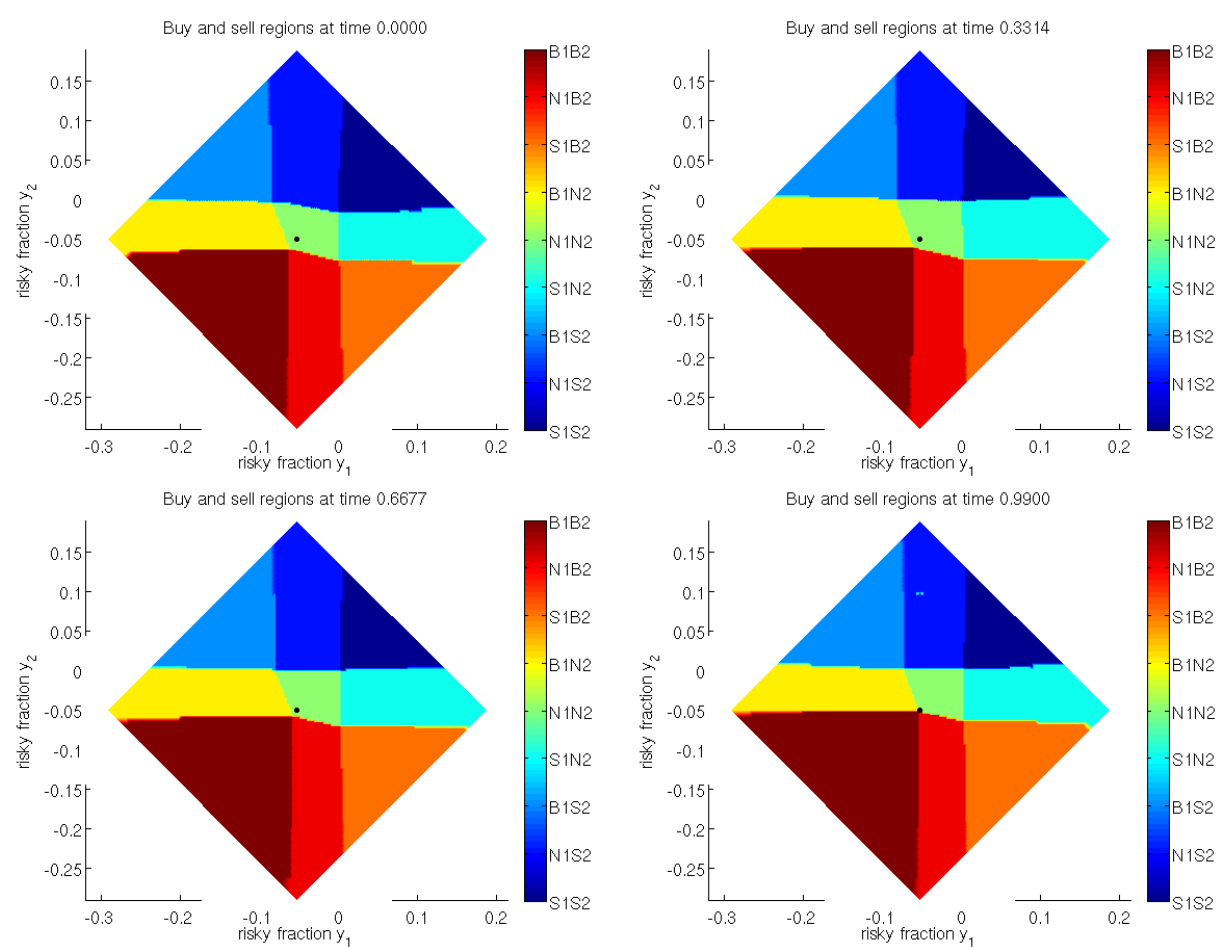

Figure 4.2. Color-coded trading regions in Example 4.1 at times near $t \in\{0,1 / 3,2 / 3,1\}$. The figure shows the major part of the reduced computational domain $\mathcal{S}_{\text {red }}$ centered at the Merton fraction (black dot).

this example with negative trend parameters which yields a Merton fraction $\hat{\eta}$ with negative positions in the stocks (short selling of stocks). This corresponds to the one-dimensional Example 3.3. Like in that example we observe in Figure 4.2 that - when approaching terminal time - the boundary of the NT-region gets closer to the Merton fraction and extends to the axes (corresponding to the red and black boundaries in Figure 3.4). This is to be expected since at terminal time we have to liquidate anyway and hence can try to get closer to the Merton fraction when we are far away while this would be too expensive if our position lies 'between' Merton fraction and 0 . Note that one year $(t=0)$ before terminal time we would still trade (sell stocks) when we have no position in the stocks, while at $t=0.6677$ this is no longer optimal. This is different in the following example.

Example 4.2. In our second example, we used the following problem data:

$$
\begin{array}{llll}
\alpha=0.3 & \text { utility exponent } & \gamma=0.5 \% & \text { trading costs } \\
\mu=\left(\begin{array}{c}
15.0 \% \\
2.0 \%
\end{array}\right) & \text { stock trends } & \sigma=\left(\begin{array}{ll}
0.42 & 0.10 \\
0.10 & 0.38
\end{array}\right) & \text { stock volatilities } \\
r=7.0 \% & \text { interest rate } & &
\end{array}
$$

on the time interval $(0,1)$. The Merton fraction is $\hat{\eta}=(1.0438,-1.0034)^{\top}$ in this example, and we consider again the case with liquidation costs. 
We ran Algorithm 4.1 with the same algorithmic parameters as in Example 4.1, but on a reduced domain of radius $R=4.0$. The algorithm used a total of 264 time steps. The evolution of time step sizes is shown in Figure 4.3. The increased number of time steps corresponds to the more pronounced movements of the trading regions, which relate to a lower level of risk aversion $(1-\alpha=0.7)$. Approximately 14 semi-smooth Newton steps were used on average per time step. In this example
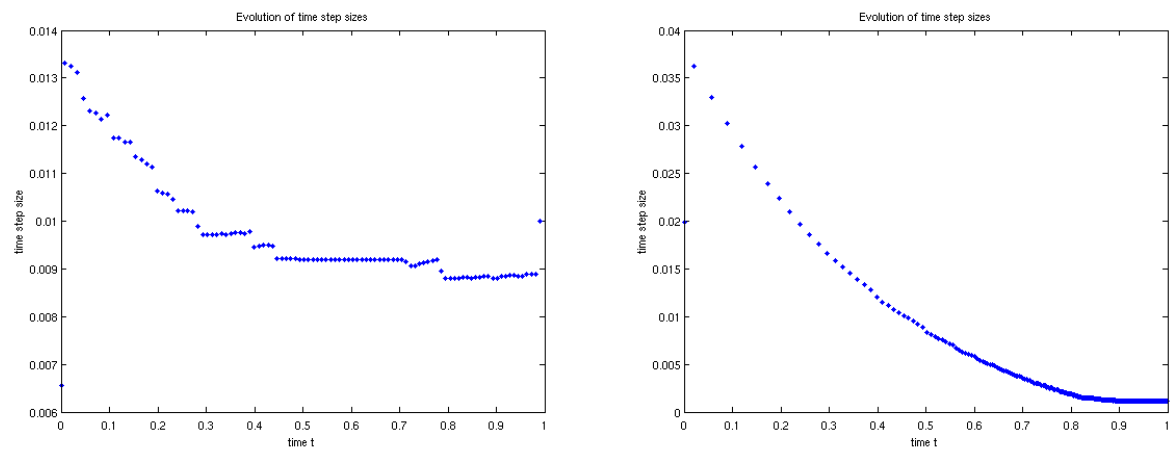

FiguRE 4.3. Evolution of time step sizes for Example 4.1 (left) and 4.2 (right).
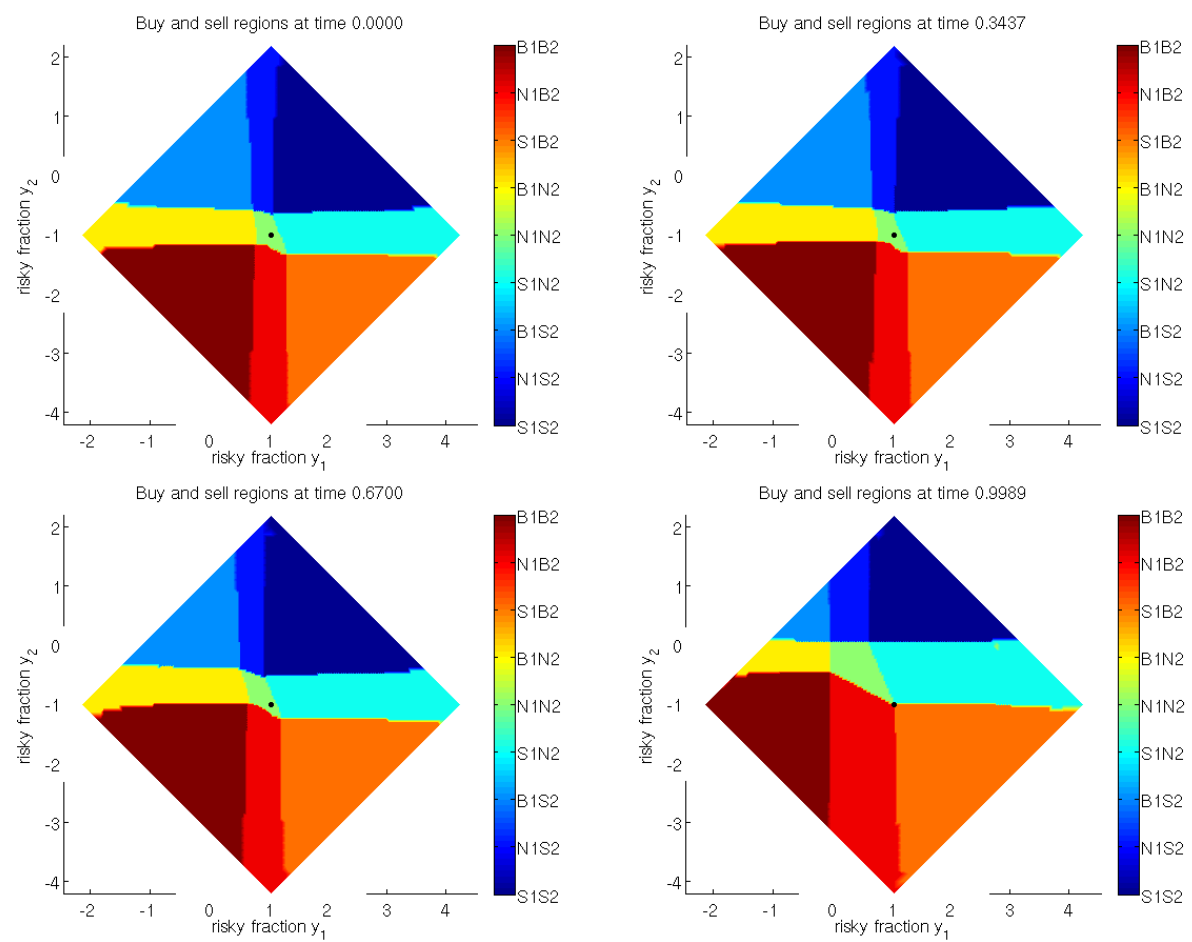

FIgURE 4.4. Color-coded trading regions in Example 4.2. The figure shows the major part of the reduced computational domain. It also shows the Merton fraction (black circle).

the first component of the Merton fraction $\hat{\eta}$ is greater than 1 (a long position for which we have to borrow money or sell the other stock short) and the second 
slightly negative (short position). The shapes of the NT-regions depending on time are similar to those in the preceding Example 4.1. The interpretation close to terminal time is the same as before. But in this example it is longer optimal to trade in the stocks when we have a position 0 in the stocks. Oppposed to Example 4.1 this is true even at $t=0.6700$ as we can see clearly from Figure 4.4. This, as well as the fact that the NT-regions are smaller at the beginning when compared to Example 4.1, is due to the lower risk aversion. Moreover the NT-region is further away from 0 as a consequence of the more extreme trend parameter of the first stock yielding a more extreme $\hat{\eta}$.

Chemnitz University of Technology, Faculty of Mathematics, D-09107 Chemnitz, GerMANY

E-mail address: roland.herzog@mathematik.tu-chemnitz.de

$U R L:$ http://www.tu-chemnitz.de/herzog

Karl Franzens University Graz, Institute for Mathematics and Scientific Computing, Heinrichstrasse 36, A-8010 Graz, Austria

E-mail address: karl.kunisch@uni-graz.at

$U R L:$ http://www.kfunigraz.ac.at/imawww/kunisch

University of Kaiserslautern, Department of Mathemaics, P.O. Box 3049, D-67663 KaisersLAUTERn, GERMANY

E-mail address: sass@mathematik.uni-kl.de

URL: http://www.mathematik.uni-kl.de/ ${ }^{\sim}$ sass/ 